\title{
Development of a multi-compartment Population Balance Model for high-shear wet granulation with Discrete Element Method
}

\author{
Kok Foong Lee ${ }^{\mathrm{a}}$, Maksym Dosta ${ }^{\mathrm{d}}$, Andrew D. McGuire ${ }^{\mathrm{a}}$, Sebastian \\ Mosbach $^{\mathrm{a}}$, Wolfgang Wagner ${ }^{\mathrm{c}}$, Stefan Heinrich ${ }^{\mathrm{d}}$, Markus Kraft*a,b \\ ${ }^{a}$ Department of Chemical Engineering and Biotechnology, University of Cambridge, \\ New Museums Site, Pembroke Street, Cambridge, CB2 3RA United Kingdom \\ corresponding author* \\ E-mail:mk306@cam.ac.uk \\ ${ }^{b}$ School of Chemical and Biomedical Engineering, Nanyang Technological University, \\ 62 Nanyang Drive, Singapore, 637459 \\ ${ }^{c}$ Weierstrass Institute for Applied Analysis and Stochastics, \\ Mohrenstraße 39, 10117 Berlin, Germany \\ ${ }^{d}$ Institute of Solids Process Engineering and Particle Technology, \\ Hamburg University of Technology, \\ Denickestrasse 15, 21073 Hamburg, Germany.
}

\begin{abstract}
This paper presents a multi-compartment population balance model for wet granulation coupled with DEM (Discrete Element Method) simulations. Methodologies are developed to extract relevant data from the DEM simulations to inform the population balance model. First, compartmental residence times are calculated for the population balance model from DEM. Then, a suitable collision kernel is chosen for the population balance model based on particle-particle collision frequencies extracted from DEM. It is found that the population balance model is able to predict the trends exhibited by the experimental size and porosity distributions by utilising the information provided by the DEM simulations.
\end{abstract}

Keywords: granulation, stochastic weighted algorithm, compartmental 
model, discrete element method, majorant kernel

\section{Introduction}

Wet granulation is a manufacturing process to produce granules with desired properties from small particles and binders, using equipments such as high-shear mixers, rotating drums and fluidised beds. Models for granulation can be broadly separated into particle level models and models which simulate the process at the unit operation level [1,2]. Models at the particle level are developed to predict inter-particle forces using fundamental physics and a review of such models can be found in [3]. At the other end of the scale, models at the unit operation level are used to predict the overall behaviour of granulation processes and this paper focuses on this aspect. Modelling approaches for wet granulation processes at the unit operation level can be loosely separated into two categories: population balance modelling and Discrete Element Method (DEM).

The population balance approach tracks the change in the particle population with time through birth and death processes: for applications in granulation, these processes are usually the coalescence and breakage of particles [4]. Traditionally, population balance models are one-dimensional with particle size as the focus. However, one-dimensional models are insufficient to describe granulation processes accurately [5]. Hence, over the last decade, population balance models published in the literature have been at least two-dimensional with liquid and solid concentrations as the properties included $[6,7,8]$ and some models also include particle pore volume $[9,10,11]$. The main advantage of the population balance approach is that it is capa- 
ble at considering detailed physical models for processes such as coalescence $[9,10,12,7]$, nucleation $[11,6]$ and breakage [13]. Population balance modelling is also suitable for long time scale studies because of its low computational effort, but it requires certain knowledge of the system in order to include the appropriate processes.

In DEM simulations, the motion of each particle is computed simultaneously using Newtonian equations of motion [14]. It is pointed out in [1] that models at the unit operation level often neglect the flow heterogeneity of powder mixing processes and the DEM approach seems to be the ideal solution to bridge this gap. However, DEM is computationally expensive and it does not consider aggregation of granules and other processes such as solidification of granules [8].

Nonetheless, it is possible to include particle flow in population balance models. This is done by dividing the simulation domain into multiple compartments with each of them having its own population balance equation. Thus, each compartment is considered to be perfectly mixed but the process rates can differ between the compartments. A main drawback of this approach is that the flux rates between the compartments are unknown but these can be determined by coupling DEM to population balance models. Population balance models that involve DEM simulations generally fall into three categories: Models that use post-processed flux rates from preliminary DEM simulations $[15,16,17,18,19]$, models that utilise DEM to develop appropriate aggregation kernels [20,21], and models that are directly coupled with DEM $[22,23,8]$. The work carried out in this paper falls into the first two categories. 
The main purpose of this paper is to improve an existing multi-compartment population balance model for a batch ploughshare mixer [24, 25] with postprocessed information from DEM simulations. Previously, the residence times of the compartments were unknown and they were tuned to fit an experimentally measured size distribution. Besides that, the existing model uses a size independent collision kernel and it is found that it is inappropriate for granulation systems [20]. In this paper, DEM simulations are performed to determine the appropriate residence times for the compartments and also to implement a size dependent collision kernel.

This paper is organised as follows: A brief description of the population balance model is given in Section 2. Then, Section 3 outlines the DEM simulations carried out in this work. Section 4 describes the stochastic particle method used to solve the population balance model, in particular the adaptation of the majorant technique $[26,27,28,29]$ to accelerate the simulation of collision events. Finally, the ability of the population balance model to predict a set of experimental results is assessed in Section 5 .

\section{Multi-compartment population balance model}

The experimental system considered in this work is the wet granulation of lactose powder with deionised water carried out in a ploughshare mixer depicted in Figure 1 and its full description can be found in [30]. It is modelled as a series of well-mixed continuous-stirred tank reactors (CSTRs) to account for spatial inhomogeneity and each reactor in the network is given a characteristic residence time, $\tau$. The configuration of the compartmental model is shown in Figure 2. As previous studies showed that radial disper- 
sion is significantly quicker compared to axial dispersion [31, 32, 33], the mixer is compartmentalised in the axial direction in the model. The multicompartment population balance model was developed in [24, 25], but the residence times of the compartments were not known and the values were tuned to fit an experimentally measured particle size distribution. In this work, the residence times of the compartments are determined using DEM and the methodology is presented in Section 3.1.

In the model, particles take positions in a domain of compartments, $\mathbb{Z}=$ $\left\{z_{1}, z_{2}, z_{3}\right\}$. Throughout this work, the residence times of $z_{1}, z_{2}$, and $z_{3}$ are denoted as $\tau_{1}, \tau_{2}$, and $\tau_{3}$ respectively. In order to capture the spreading of binder liquid which is often regarded as a crucial stage in granulation processes $[34,3,35]$, the middle compartment $z_{2}$ is defined as the spray zone where liquid addition occurs. With the exception of liquid addition, each compartment simulates the same particle processes described in Section 2.2, but at different rates to capture the spatial inhomogeneity of the process.

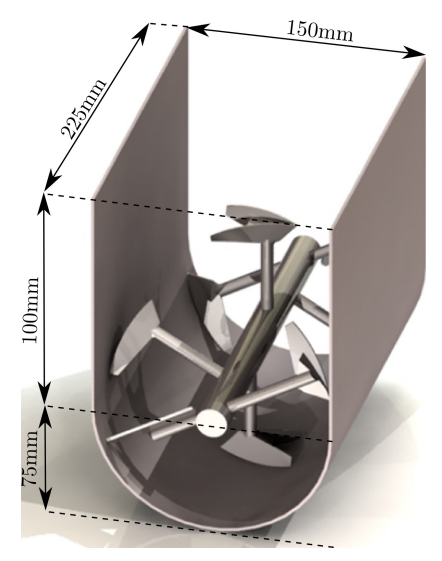

Figure 1: CAD drawing of the mixer. The radial direction refers to direction in which the blades rotate and the axial direction refers to the direction along the shaft. 


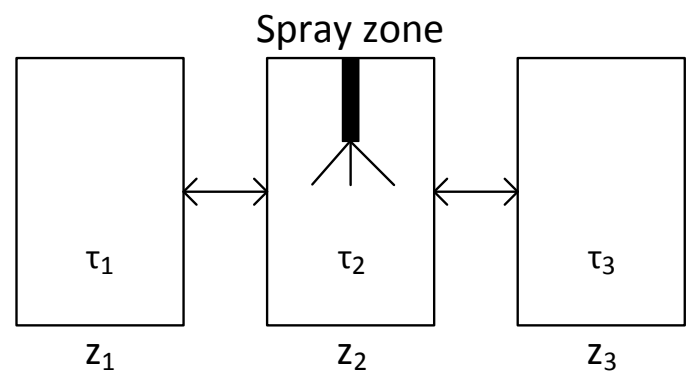

Figure 2: Configuration of the compartmental model. The mixer (Figure 1) is compartmentalised in the axial direction. Each compartment has the same size.

\subsection{Type-space}

The type-space is the mathematical description of a particle. In this model, the type-space $\mathbb{X}=\left\{s_{\mathrm{o}}, s_{\mathrm{r}}, l_{\mathrm{e}}, l_{\mathrm{i}}, p\right\}$ has five independent nonnegative variables which describe a granule. They are original solid volume $s_{\mathrm{o}}$, reacted solid volume $s_{\mathrm{r}}$, external liquid volume $l_{\mathrm{e}}$, internal liquid volume $l_{\mathrm{i}}$, and pore volume $p$.

For a particle of type $x=\left(s_{\mathrm{o}}, s_{\mathrm{r}}, l_{\mathrm{e}}, l_{\mathrm{i}}, p\right)$, the following derived properties are defined in terms of its internal variables:

- Particle volume: The volume is calculated as:

$$
v(x)=s_{\mathrm{o}}+s_{\mathrm{r}}+l_{\mathrm{e}}+p .
$$

- Size: Assuming that the particles are spherical, the radius is:

$$
R(x)=\sqrt[3]{\frac{3}{4 \pi} v(x)}
$$

and the diameter $d(x)$ is simply $2 R(x)$. 
- Mass: Assuming that the densities of the liquids and the reacted solids are the same:

$$
\rho_{l_{\mathrm{e}}}=\rho_{l_{\mathrm{i}}}=\rho_{s_{\mathrm{r}}}
$$

the particle mass is calculated as:

$$
m(x)=\rho_{s_{\mathrm{o}}} \times s_{\mathrm{o}}+\rho_{l_{\mathrm{e}}} \times\left[s_{\mathrm{r}}+l_{\mathrm{i}}+l_{\mathrm{e}}\right],
$$

where $\rho_{s_{\mathrm{o}}}$ and $\rho_{l_{\mathrm{e}}}$ are input parameters. Further explanation regarding this assumption is given at the end of Section 4 .

- Porosity:

$$
\epsilon(x)=\frac{p}{v(x)} .
$$

- External surface area $a_{\mathrm{e}}$ and internal surface area $a_{\mathrm{i}}$ : Assuming spherical shape:

$$
\begin{aligned}
& a_{\mathrm{e}}=\pi^{1 / 3}(6 v(x))^{2 / 3} \\
& a_{\mathrm{i}}=C p^{2 / 3} .
\end{aligned}
$$

The constant $C$ is an input parameter. Higher values of $C$ indicate more tortuous pores. The value $C=15$ which gave good results in [4] is used.

\subsection{Particle processes}

In this model, particles may be created and evolved through several different processes. Most of the processes are described briefly here. For the full 
description of the model and the reasoning behind the submodels, readers can refer to $[4,36,37,38,39,40]$.

Liquid addition: Liquid droplets are introduced into the particle ensemble in the form of

$$
x_{\mathrm{droplet}}=\left(0,0, l_{\mathrm{e}}, 0,0\right)
$$

at the rate

$$
r_{z, \text { droplet }}=\left\{\begin{aligned}
\frac{\dot{V}_{1}}{V_{\text {droplet }}} & , \text { if } z=z_{2} \\
0 & , \text { otherwise }
\end{aligned}\right.
$$

where $\dot{V}_{\mathrm{l}}$ is the volumetric flow rate of the binder (experimental condition) and $V_{\text {droplet }}$ is the droplet volume.

Breakage: The current model considers a binary breakage process [36, 37]:

$$
\left(x_{i}\right) \rightarrow\left(x_{j}\right),\left(x_{i}-x_{j}\right),
$$

and each particle $x_{i}$ breaks at the frequency

$$
r_{z, \text { break }}\left(x_{i}\right)=\left\{\begin{aligned}
k_{z, \text { break }} U_{\mathrm{imp}}^{2}\left(p+l_{\mathrm{e}}\right) & , \text { if } v\left(x_{i}\right) \geq v_{\text {parent,min }} \\
0 & , \text { otherwise }
\end{aligned}\right.
$$

with input parameters $k_{z \text {, break }}$ (breakage rate constant which may differ in different compartments) and $U_{\text {imp }}$ (impact velocity between the particles and impeller blades). $v_{\text {parent,min }}$ is the smallest parent particle that can undergo breakage. 
Chemical reaction: The liquid components $\left(l_{\mathrm{e}}\right.$ and $\left.l_{\mathrm{i}}\right)$ are transformed into reacted solid, $s_{\mathrm{r}}$, at the rates

$$
\begin{array}{ll}
r_{z, \text { reac }, \mathrm{e}}(x)=k_{z, \text { reac }} a_{\mathrm{e}}(x) \frac{l_{\mathrm{e}}}{l_{\mathrm{e}}+s_{\mathrm{r}}}, & \text { for } l_{\mathrm{e}} \rightarrow s_{\mathrm{r}}, \\
r_{z, \text { reac }, \mathrm{i}}(x)=k_{z, \text { reac }} a_{\mathrm{i}}(x) \frac{l_{\mathrm{i}}}{l_{\mathrm{i}}+s_{\mathrm{r}}}, & \text { for } l_{\mathrm{i}} \rightarrow s_{\mathrm{r}},
\end{array}
$$

where $a_{\mathrm{e}}$ and $a_{\mathrm{i}}$ are the external and internal surface areas respectively. $k_{z \text {, reac }}$ is the reaction rate constant which may differ in different compartments. This work studies the granulation of lactose powder with deionised water. Without the presence of lactase, the only reaction is the hydration of lactose by water [41].

Penetration: This process refers to the diffusion of external liquid into the pores, i.e. transformation of $l_{\mathrm{e}}$ into $l_{\mathrm{i}}$, and occurs at the rate

$$
r_{z, \text { pen }}(x)=k_{z, \text { pen }} \eta^{-0.5} l_{\mathrm{e}}\left(p-l_{\mathrm{i}}\right),
$$

where $k_{z \text {, pen }}$ is the penetration rate constant which may differ in different compartments and $\eta$ is the viscosity of the binder.

Particle transport: Each particle leaves its current compartment $z$ at the rate

$$
r_{z, \text { transport }}=\frac{1}{\tau_{z}},
$$

where $\tau_{z}$ denotes the residence time of compartment $z$.

Collision: The rate of collision between the particles $x_{i}$ and $x_{j}$ is defined by the kernel:

$$
K_{z}\left(x_{i}, x_{j}\right)=\omega_{\text {impeller }} k_{z, \text { col }} C\left(d_{i}, d_{j}\right),
$$


where $\omega_{\text {impeller }}$ is the impeller speed, $k_{z, \text { col }}$ is the collision rate constant which may differ in different compartments, and $C\left(d_{i}, d_{j}\right)$ is the collision rate function which defines the collision frequency between particles with diameters $d_{i}$ and $d_{j}$. It is assumed that the collision rate function is independent of the impeller speed. In the original model $[4,36,37]$, the collision kernel is independent of size and the collision rate function is first introduced in this work. In Section 3.1, a suitable size dependent collision rate function is determined with collision rates extracted from a DEM simulation.

Each collision leads to the compaction of the colliding particles and the change in porosity is described by

$$
\Delta \epsilon(x)= \begin{cases}k_{z, \text { comp }}\left(\epsilon(x)-\epsilon_{\min }\right) & , \text { if } \epsilon(x) \geq \epsilon_{\min } \\ 0, & \text { otherwise }\end{cases}
$$

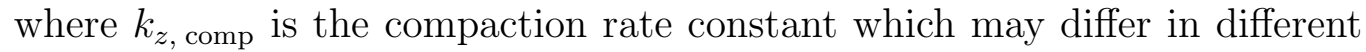
compartments and $\epsilon_{\min }$ is the minimum porosity. However, the success of coalescence is controlled by the collision efficiency $\widetilde{K}$ calculated based on the Stokes criterion $[42,36]$ which compares the kinetic energy of collision to the dissipation energy provided by the binder:

$$
\widetilde{K}\left(x_{i}, x_{j}\right)= \begin{cases}1, & \text { (successful coalescence }) \\ 0, & \text { (unsuccessful coalescence })\end{cases}
$$

Depending on the value of $\widetilde{K}\left(x_{i}, x_{j}\right)$, a collision between the particles $x_{i}$ and $x_{j}$ has the following outcomes

$$
\begin{aligned}
\left(x_{i}\right),\left(x_{j}\right) \rightarrow T_{\mathrm{comp}}\left(x_{i}+x_{j}\right), & \text { if } \widetilde{K}\left(x_{i}, x_{j}\right)=1, \\
\left(x_{i}\right),\left(x_{j}\right) \rightarrow T_{\mathrm{comp}}\left(x_{i}\right), T_{\mathrm{comp}}\left(x_{j}\right), & \text { if } \widetilde{K}\left(x_{i}, x_{j}\right)=0,
\end{aligned}
$$


where $T_{\text {comp }}$ represents the compaction process (16).

The coalescence efficiency $\widetilde{K}$ is a function of the viscous Stokes number and the critical Stokes number, with

$$
\widetilde{K}\left(x_{i}, x_{j}\right)=\left\{\begin{array}{l}
1, \text { if } e_{\text {coag }}\left(x_{i}, x_{j}\right)=0, \\
0, \text { if } e_{\text {coag }}\left(x_{i}, x_{j}\right)>0 \text { and } \mathrm{St}_{\mathrm{v}}^{*}\left(x_{i}, x_{j}\right) \geq \mathrm{St}_{\mathrm{v}}\left(x_{i}, x_{j}\right), \\
0, \text { otherwise. }
\end{array}\right.
$$

The coefficient of restitution is defined as the geometric average of the coefficients of restitution of the single particles $x_{i}$ and $x_{j}$,

$$
e_{\mathrm{coag}}\left(x_{i}, x_{j}\right)=\sqrt{e\left(x_{i}\right) \cdot e\left(x_{j}\right)} .
$$

The coefficient of restitution of each particle is

$$
e(x)=\left\{\begin{aligned}
0.2, & \text { if } s_{\mathrm{o}}+s_{\mathrm{r}}>0, \\
0, & \text { otherwise } x=x_{\text {droplet }} .
\end{aligned}\right.
$$

The value 0.2 is obtained from experimental measurements and further details of the measurements can be found in Section 3.

The viscous Stokes number is computed as

$$
\mathrm{St}_{\mathrm{v}}\left(x_{i}, x_{j}\right)=\frac{\widetilde{m}\left(x_{i}, x_{j}\right) U_{\mathrm{col}}}{24 \pi \eta \widetilde{R}\left(x_{i}, x_{j}\right)^{2}},
$$

with input parameters $U_{\text {col }}$ (collision velocity) and $\eta$ (binder viscosity). The collision velocity is calculated as $10 \%$ of the impeller speed [37]:

$$
U_{\text {col }}=0.1\left(2 \pi \omega_{\text {impeller }} r_{\text {impeller }}\right)
$$

At the operating conditions used in this paper, the value derived from the DEM simulations is very close to the value calculated with Equation (20). 
The harmonic mass of $x_{i}$ and $x_{j}$ is

$$
\widetilde{m}\left(x_{i}, x_{j}\right)=\frac{2 m\left(x_{i}\right) m\left(x_{j}\right)}{m\left(x_{i}\right)+m\left(x_{j}\right)} .
$$

The harmonic radius computes as

$$
\widetilde{R}\left(x_{i}, x_{j}\right)=\frac{2 R\left(x_{i}\right) R\left(x_{j}\right)}{R\left(x_{i}\right)+R\left(x_{j}\right)} .
$$

The critical Stokes number is defined by

$$
\mathrm{St}_{\mathrm{v}}^{*}\left(x_{i}, x_{j}\right)=\left(1+\frac{1}{e_{\text {coag }}\left(x_{i}, x_{j}\right)} \ln \left(\frac{h_{\text {binder }}\left(x_{i}, x_{j}\right)}{h_{\mathrm{a}}},\right)\right)
$$

with the input parameter $h_{\mathrm{a}}=2.09 \times 10^{-7} \mathrm{~m}$ (characteristic length scale of surface asperities, within the range measured in [45]). The thickness of the binder layer $h_{\text {binder }}\left(x_{i}, x_{j}\right)$ is defined as the combined binder thickness of the particles $x_{i}$ and $x_{j}$

$$
h_{\text {binder }}\left(x_{i}, x_{j}\right)=\frac{h_{\text {binder }}\left(x_{i}\right)+h_{\text {binder }}\left(x_{j}\right)}{2},
$$

with the thickness of the binder layer of a particle with the properties $x$ calculated by

$$
h_{\text {binder }}(x)=\frac{1}{2} \sqrt[3]{\frac{6}{\pi}}\left[\sqrt[3]{v(x)}-\sqrt[3]{v(x)-l_{\mathrm{e}}}\right]
$$

\section{DEM}

The DEM implementation in this paper uses the Hertz-Mindlin model for the calculations of both the particle-particle and particle-wall contact. For more details regarding the implementation, readers can refer to [43]. 
The geometry used in the DEM simulations is constructed to match the laboratory mixer and the CAD drawing is shown in Figure 1.

Dry spherical particles are used in the simulations and the effects of the binder liquid are ignored. DEM simulations are carried out in this work to provide two crucial information needed by the population balance model. The first piece of information is the residence times of the compartments and the second piece of information is the collision rates between particles as a function of size. The computational cost of a DEM simulation varies according to the simulation time required, number of particles and the range of particle size. In order to obtain reliable estimates of the residence times, it is necessary to perform the simulation for a long period of time. On the other hand, it is required to include a wide range of particle sizes to estimate the collision rate function but long simulation time is unnecessary. Therefore, two separate simulations with different specifications are performed to balance between what is achievable within reasonable computational costs and the quality of data obtainable.

The first DEM simulation is performed to estimate the residence times of the compartments. Uniformly sized large particles $(2.6 \mathrm{~mm})$ are used so that it is possible to simulate the system long enough. The number of particles is adjusted to match the bed volume in the experiments and this results in approximately 80,000 particles. In Section 3.1, it will become evident that a long simulation time is necessary to obtain enough data points to estimate the residence times accurately.

The second DEM simulation is carried out to estimate the collision rate function. The collision frequency between a pair of particle sizes is considered 
a single data point. Therefore, a wide range of particle sizes is necessary for this simulation to obtain sufficient data points to estimate the collision rate function. Since it is unnecessary to simulate the system for that long, it is also possible to increase the number of particles in the expense of $\mathrm{CPU}$ time for this simulation. The particles for this simulation are generated according to a measured size distribution obtained from the same experimental conditions described later in Section 5. To limit the number of particles, it is required to apply a lower cut off size of $1 \mathrm{~mm}$ and this resulted in approximately 200,000 particles. The reduced size distribution used in this DEM simulation is shown in Figure 3.

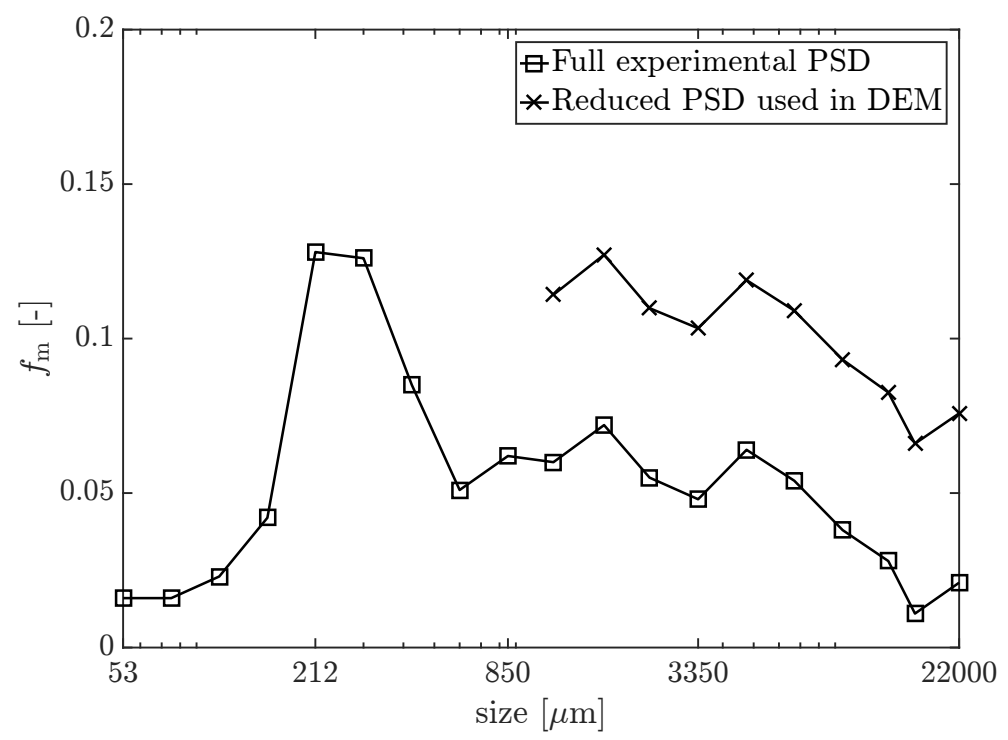

Figure 3: Reduced particle size distribution used in the DEM simulation compared to the experimental size distribution.

Figure 4 shows the initial states of the simulations. For both cases, the number of particles is adjusted to approximate the experimental bed volume. 


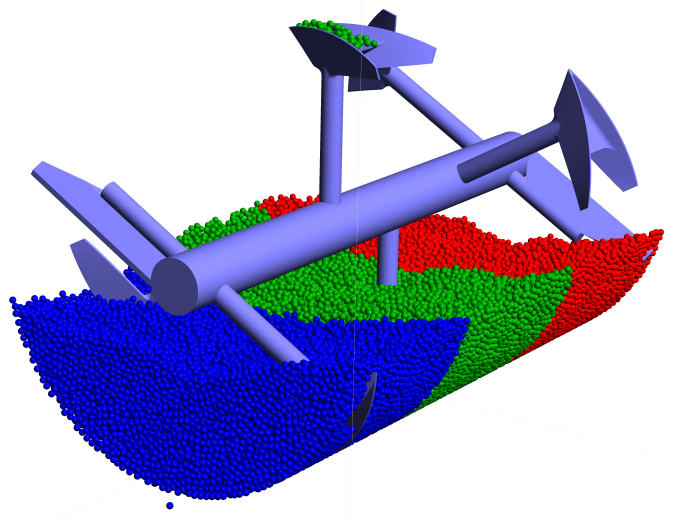

(a) DEM with uniform particles.

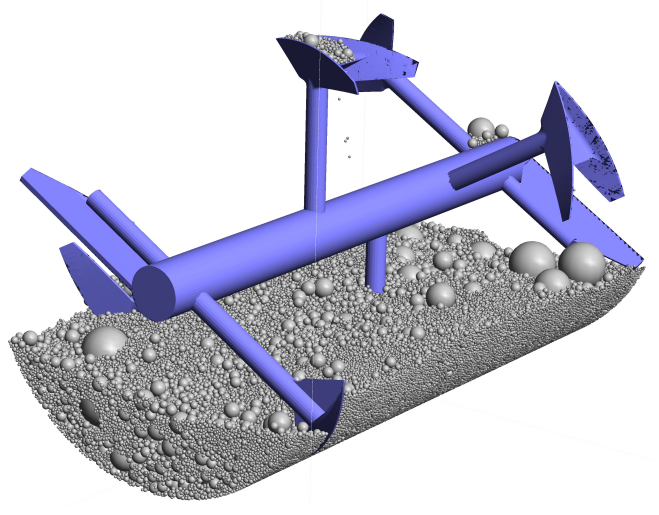

(b) DEM with particle size distribution.

Figure 4: Initial states of the DEM simulations. Particles are tagged with colours [red, green, blue] for the uniform case to determine the residence times in Section 3.1.

Both case studies are simulated at the same impeller speed (2 rev/s) as the experimental system we are modelling (described later in Section 5) and the material properties used for the simulations are summarised in Table 1. Among the properties, the density, coefficient of restitution and Young's Modulus are experimentally measured, and the rest are extracted from the literature. Dried lactose granules are used for the measurements and the effects of the binder are ignored. The coefficient of restitution of dried lactose granules is measured using an optical technique described in [44] and an average value of 0.2 is obtained from the measurements. In the experiments, a high-speed camera was used to record the impact and rebound of the granules as they were dropped onto a glass plate. The coefficient of restitution of the granules were determined by comparing the impact and rebound velocities from the images. 
The Young's Modulus is calculated from single granule compression tests described in [45]. An average value of $1.7 \times 10^{7} \mathrm{~Pa}$ is used for the simulation with uniform particles and the values used in the simulation with the experimental particle size distribution are shown in Figure 5 where the Young's Modulus is set according to the particle size.

Table 1: Material properties used in DEM. Values shown are for both simulations with uniform particle and PSD unless otherwise stated.

\begin{tabular}{lc}
\hline Property & Value \\
\hline Density of lactose & $1545 \mathrm{~kg} \mathrm{~m}^{-3}$ \\
Coefficient of restitution & 0.2 \\
Coefficient of friction (particle-particle) & 0.2 \\
Coefficient of friction (particle-wall) & 0.35 \\
Poisson ratio [46] & 0.3 \\
Young's Modulus of lactose (uniform size) & $1.7 \times 10^{7} \mathrm{~Pa}$ \\
Young's Modulus of lactose (PSD) & Refer Figure 5 \\
Young's Modulus of steel [46] & $2.15 \times 10^{11} \mathrm{~Pa}$ \\
\hline
\end{tabular}

\subsection{Compartmental residence times}

This section describes the methodology to estimate the residence times of the compartments using the DEM simulation with uniform particles (Figure 4a). The easiest way to calculate the residence time for a compartment is to evaluate the average duration a particle spends in the compartment before it exits the compartment. However, this approach is not suitable to 


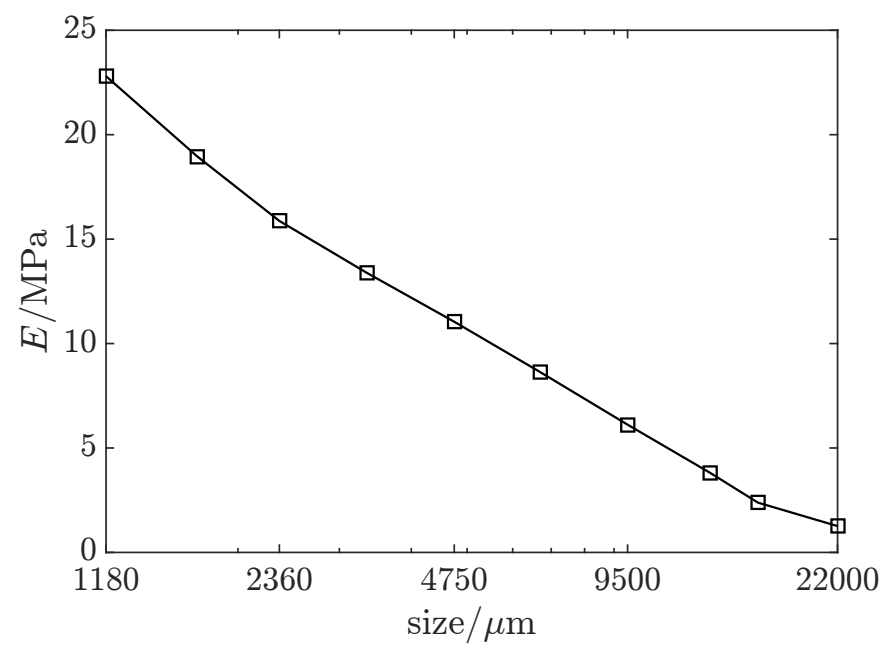

Figure 5: Young's Modulus, E, used in the DEM simulation for the estimation of the collision rate function.

derive representative residence times for the compartments because the frequency distribution of residence times is very wide as shown in Figure 6 for the compartment $z_{1}$. To obtain the frequencies for the respective residence times, each time a particle passes through the boundary between $z_{1}$ and $z_{2}$, the time spent by the particle in $z_{1}$ before returning to $z_{2}$ is recorded. The enormous frequencies recorded for short residence times are contributed by the particles oscillating at the border between $z_{1}$ and $z_{2}$. From the plot, it can also be observed that there are particles which stayed in the same compartment for almost the entire simulation (system is simulated for 200 seconds). Therefore, it is not possible to calculate a reliable average residence time by tracking the actual residence times of the particles.

Instead, the compartmental residence times are estimated by considering the evolution of particle concentrations in the compartments and assuming 


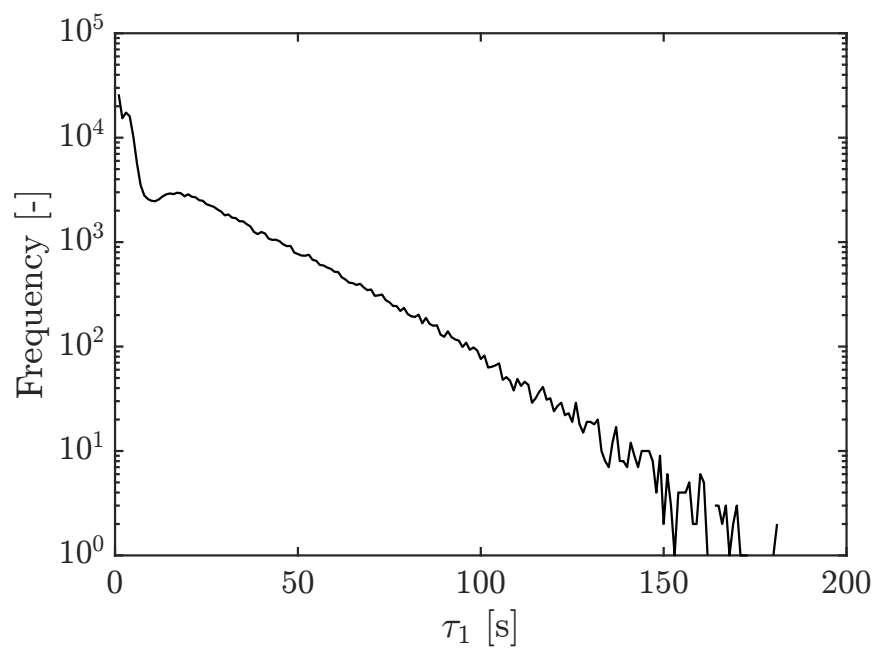

Figure 6: Residence time distribution for the compartment $z_{1}$.

each compartment is well-mixed. This is done by tagging the particles in each compartment with colours $\left(z_{1}\right.$ : red, $z_{2}$ : green, $z_{3}$ : blue) as shown in Figure $4 \mathrm{a}$ at the beginning of the simulation. Then, the volume fraction of the colour $c$ in compartment $z$ at time $t$ is calculated by

$$
\hat{\nu}_{z, c}(t)_{\mathrm{DEM}}=\frac{V_{\text {particle }}(t, z, c)}{\sum_{c} V_{\text {particle }}(t, z, c)},
$$

where $V_{\text {particle }}(t, z, c)$ is the total volume of particles of the colour $c$ in compartment $z$ at time $t$ and $\sum_{c} V_{\text {particle }}(t, z, c)$ is the total volume of all the particles which is located in compartment $z$ at time $t$.

The evolutions of the volume fractions in each compartment are fitted to a model which assumes an idealised reactor network. In the model, each compartment is assumed to be perfectly mixed with a tertiary particle colour system initially loaded as [red, green, blue]. It assumes that the separation between compartment boundaries is sufficiently large and the time step between analysis frames is sufficiently small such that particles cannot 'skip' 
over compartments (e.g. from $z_{1}$ directly to $z_{3}$ ). The model assumes constant mass, constant particle density and constant particle size in each compartment hold-up throughout the mixing process. The model was solved for the evolution of particle volume fraction $\hat{\nu}$ for each colour $c$ in each compartment $z$ to yield four unique model equations:

$$
\begin{aligned}
\hat{\nu}_{1, \text { red }}(t)_{\text {model }} & =\left[\frac{1}{2} \exp \left(-\frac{t}{\tau_{1}}\right)+\frac{1}{6} \exp \left(-\frac{3 t}{\tau_{1}}\right)-\frac{2}{3}\right], \\
\hat{\nu}_{2, \text { red }}(t)_{\text {model }} & =\left[-\frac{1}{3} \exp \left(-\frac{3 t}{\tau_{1}}\right)+\frac{1}{3}\right], \\
\hat{\nu}_{3, \text { red }}(t)_{\text {model }} & =\left[-\frac{1}{2} \exp \left(-\frac{t}{\tau_{1}}\right)+\frac{1}{6} \exp \left(-\frac{3 t}{\tau_{1}}\right)+\frac{1}{3}\right], \\
\hat{\nu}_{2, \text { green }}(t)_{\text {model }} & =\left[\frac{2}{3} \exp \left(-\frac{3 t}{\tau_{1}}\right)-\frac{2}{3}\right],
\end{aligned}
$$

similar to the residence times, the subscripts 1, 2, and 3 correspond to the compartments $z_{1}, z_{2}$, and $z_{3}$.

Due to the symmetrical nature of both the mixture operation and loading it is known that in an ideal system:

$$
\begin{aligned}
\hat{\nu}_{1, \text { blue }}(t) & =\hat{\nu}_{3, \text { red }}(t), \\
\hat{\nu}_{2, \text { blue }}(t) & =\hat{\nu}_{2, \text { red }}(t), \\
\hat{\nu}_{3, \text { blue }}(t) & =\hat{\nu}_{1, \text { red }}(t), \\
\hat{\nu}_{1, \text { green }}(t)=\hat{\nu}_{3, \text { green }}(t) & =\hat{\nu}_{2, \text { red }}(t)=\hat{\nu}_{2, \text { blue }}(t),
\end{aligned}
$$

and

$$
\tau_{1}=2 \tau_{2}=\tau_{3}
$$

Equations (22 - 30) form a complete set of equations to describe the volume fraction evolution of each particle colour in each compartment of the ideal 
system. For the full derivation of the model, readers can refer to Appendix A.

The compartmental residence times are then determined by fitting the volume fractions measured from the DEM simulation to the ideal model and Figure 7 shows the results for the compartment $z_{1}$. The results for the other compartments are determined in a similar manner and they are summarised in Table 2. The average value, $\tau_{1}=56 \mathrm{~s}$, is used for the compartmental population balance model. The values of $\tau_{2}$ and $\tau_{3}$ are determined according to Equation (30).

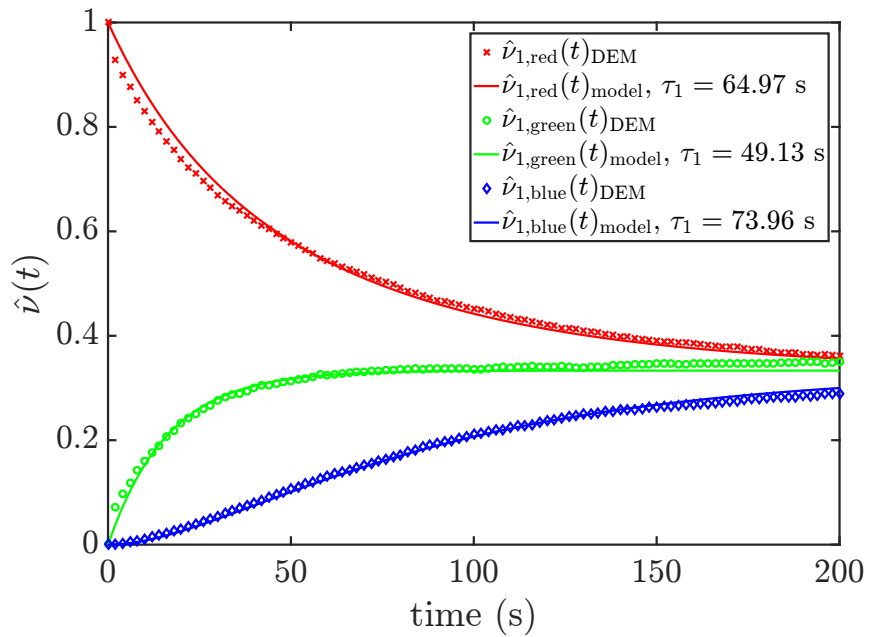

Figure 7: Ideal model fitted to the measured volume fractions in $z_{1}$.

\subsection{Collision rate function}

Results presented in this section are obtained using the DEM simulation carried out with the experimental particle size distribution (Figure 4b). As mentioned in Section 2.2, DEM is used to determine a suitable collision rate 
Table 2: Fitted residence times.

\begin{tabular}{cccc}
\hline compartment, $z$ & particle type, $c$ & $\hat{\nu}_{z, c}(0)$ & $\tau_{1}(\mathrm{~s})$ \\
\hline 1 & red & 1.0 & 64.97 \\
1 & green & 0.0 & 49.13 \\
1 & blue & 0.0 & 73.96 \\
\hline 2 & red & 0.0 & 45.10 \\
2 & green & 1.0 & 51.44 \\
2 & blue & 0.0 & 60.52 \\
\hline 3 & red & 0.0 & 70.61 \\
3 & green & 0.0 & 33.09 \\
3 & blue & 1.0 & 55.12 \\
\hline & & & $\overline{\tau_{1}} \approx 56$
\end{tabular}

function $C\left(d_{i}, d_{j}\right)$ for the population balance model. This is done by fitting the collision frequencies extracted from the DEM simulation to a list of known collision rate functions in the literature.

The collision frequency normalised by the number of particles in DEM is calculated as [20]:

$$
C\left(d_{i}, d_{j}\right)=\frac{N_{\mathrm{col}}\left(d_{i}, d_{j}\right)}{N_{\mathrm{part}}\left(d_{i}\right) N_{\mathrm{part}}\left(d_{j}\right) \Delta t},
$$

where $N_{\text {col }}\left(d_{i}, d_{j}\right)$ is the number of collisions between particles with the diameters $d_{i}$ and $d_{j}$ during the time interval $\Delta t$, and $N_{\text {part }}\left(d_{i}\right)$ is the number of particles with diameter $d_{i}$. In this case, particle-particle collisions are recorded over a period of 1 second and a total of $2.8 \times 10^{7}$ collisions are considered. 
Table 3: Collision rate functions in the literature. Assembled by [20].

\begin{tabular}{cc}
\hline Name & Collision rate function \\
\hline Size independent [4] & $C\left(d_{i}, d_{j}\right)=1$ \\
Equipartition of kinetic energy (EKE) [21] & $C\left(d_{i}, d_{j}\right) \propto\left(d_{i}+d_{j}\right)^{2} \sqrt{\frac{1}{d_{i}{ }^{3}}+\frac{1}{d_{j}{ }^{3}}}$ \\
Equipartition of translational momentum (ETM) [47] & $C\left(d_{i}, d_{j}\right) \propto\left(d_{i}+d_{j}\right)^{2} \sqrt{\frac{1}{d_{i}{ }^{6}}+\frac{1}{d_{j}{ }^{6}}}$ \\
Perikinetic or Brownian motion [20] & $C\left(d_{i}, d_{j}\right) \propto \frac{d_{i}+d_{j}}{d_{i} d_{j}}$ \\
Orthokinetic or induced shear [20] & $C\left(d_{i}, d_{j}\right) \propto\left(d_{i}+d_{j}\right)^{3}$ \\
\hline
\end{tabular}

Each of the rate functions in Table 3 is fitted to the frequencies calculated from DEM and the results are plotted in Figure 8. Figure 8a shows the collision frequencies in DEM calculated with Equation (31) and it can be observed that the majority of collisions in the simulation are between the large and small particles. Particles of similar sizes collide with each other much less frequently than with particles of different sizes, as shown by the drop in collision frequencies along the axis of symmetry. Figures $8 \mathrm{~b}-8 \mathrm{f}$ show the collision rate functions in Table 3 fitted to the normalised frequencies calculated from DEM. From these plots, the collision rate functions which show similar patterns are the EKE and ETM kernels (Figures 8c and 8d).

In order to decide between the EKE and ETM kernels, their relative errors with respect to the collision frequencies from DEM are compared. For each 
data point available, the relative error is calculated as:

$$
\epsilon_{\mathrm{rel}}\left(d_{i}, d_{j}\right)=\frac{\left|C_{\mathrm{DEM}}\left(d_{i}, d_{j}\right)-C_{\mathrm{EKE} / \mathrm{ETM}}\left(d_{i}, d_{j}\right)\right|}{C_{\mathrm{DEM}}\left(d_{i}, d_{j}\right)} .
$$

Figure 9 compares the relative errors for the EKE and ETM rate functions compared to the DEM extracted frequencies. The plots here show the pointby-point comparison between the DEM extracted frequencies in Figure 8a with the values in Figures 8c (EKE) and 8d (ETM). Based on the plots, it can be observed that the ETM rate function completely fails to predict the collision frequencies as the relative errors for most of the points exceed 1. For the EKE rate function, the average relative error over the points considered is 0.38 . Considering that particles below $1 \mathrm{~mm}$ are ignored in the DEM simulation and the various idealised assumptions made in the EKE rate function, the overall fit of the EKE rate function to the collision frequencies from DEM can be considered excellent.

\section{Numerical method for the population balance model}

As the population balance model is five-dimensional, the stochastic approach is the only viable method to solve it. There are two popular stochastic particle methods, which are the Direct Simulation Algorithm (DSA) and the Stochastic Weighted Algorithm (SWA) [25, 24, 48, 27, 26, 49, 50, 51]. In DSA, each computational particle represents the same number of real particles. SWA differs from DSA in that a statistical weight is attached to each computational particle and this weight is proportional to the number of real particles represented by the computational particle. As pointed out in $[24,25]$, DSA is unsuitable for compartmental models because of the high statistical noise observed, hence only SWA is used in this work. 
For the population balance model described in Section 2, each compartment $z$ is simulated with a system of stochastic particles:

$$
Q_{z}(t)=\left(z, x_{i}, w_{i}\right), \quad i=1, \ldots, N_{z}(t)
$$

where $z \in \mathbb{Z}, x_{i} \in \mathbb{X}, w_{i} \in\left(0, w_{\max }\right]$ is the statistical weight and $N_{z}(t)$ is the number of computational particles at time $t$. Each compartment $z$ is scaled by a normalisation parameter $n_{z}$ and it can be interpreted as the computational sample volume [25]. The number concentration of the particles of type $x_{i}$ located in $z$ is given by $w_{i} / n_{z}$.

The sequence of random processes (33) approximates the concentration measures $c(t, z, \mathrm{~d} x)$ as $n_{z} \rightarrow \infty$ :

$$
\frac{1}{n_{z}} \sum_{i=1}^{N_{z}(t)} w_{i} \varphi\left(z, x_{i}\right) \underset{n_{z} \rightarrow \infty}{\longrightarrow} \int_{\mathbb{X}} \varphi(z, x) c(t, z, \mathrm{~d} x),
$$

where $\varphi$ is some suitable test function. Further details regarding the convergence properties of the stochastic algorithm can be found in [27, 25]. The normalisation parameter $n_{z}$ is initialised such that

$$
\frac{1}{n_{z}} \sum_{i=1}^{N_{z}(0)} w_{i} \approx \int_{\mathbb{X}} c(0, z, \mathrm{~d} x) \approx \frac{N_{z}(0)}{N_{\text {real }}}
$$

$N_{\text {real }}=6.5 \times 10^{9}$ is the approximated number of real particles in the system. As the parameter $N_{\text {real }}$ only affects the value $n_{z}$, the choice for this value is not significant because $n_{z}$ is merely a scaling parameter for the stochastic algorithm.

The number of initialised particles, $N_{z}(0)$, is set at $0.75 N_{\max }$. The parameter $N_{\max }$ controls the maximum number of particles in each compartment by rescaling the ensemble when the ensemble is full [25]. 
The particle processes described in Section 2.2 are either implemented as jump processes or continuous processes. For this model, the jump processes include liquid addition, collision, breakage and particle transport, whereas the continuous processes include chemical reaction and penetration. In this section, the jump mechanisms and rates in the context of SWA for each of the particle processes are described, followed by the overall simulation algorithm.

\subsection{Liquid addition}

At the rate given by

$$
R_{z, \text { droplet }}= \begin{cases}3 \times \frac{n_{z}}{V_{\text {reactor }}} \frac{\dot{V}_{1}}{V_{\text {droplet }}} \quad, & \text { for } z=z_{2}, \\ 0 \quad & \text { for } z=z_{1}, z_{3},\end{cases}
$$

liquid particles of the form $\left(z, x_{\text {droplet }}, 1\right)$ are introduced into the system. $V_{\text {reactor }}$ is the volume of the mixer and the term $n_{z} / V_{\text {reactor }}$ scales the rate according to the normalisation parameter.

\subsection{Breakage}

The total breakage jump rate in a compartment $z$ is

$$
R_{z, \text { break }}=\sum_{i=1}^{N_{z}(t)} r_{z, \text { break }}\left(x_{i}\right) .
$$

The index $i$ of the particle to break within a compartment $z$ is selected with probability

$$
\frac{r_{z, \text { break }}\left(x_{i}\right)}{R_{z, \text { break }}}
$$

and the jump process takes the following form

$$
\left(z, x_{i}, w_{i}\right) \rightarrow\left(z, y, \gamma_{\text {break }}\left(x_{i}, w_{i}, y\right)\right)
$$


The fragment $y$ takes the following forms with different probabilities:

$$
\begin{aligned}
& y=x_{j} \quad, \quad \text { with probability }=\frac{m\left(x_{j}\right)}{m\left(x_{i}\right)}, \\
& y=x_{i}-x_{j} \quad, \quad \text { with probability }=1-\frac{m\left(x_{j}\right)}{m\left(x_{i}\right)} .
\end{aligned}
$$

The breakage weight transfer function $\gamma_{\text {break }}$ is defined to conserve total mass:

$$
\gamma_{\text {break }}\left(x_{i}, w_{i}, y\right)=w_{i} \times \frac{m\left(x_{i}\right)}{m(y)} .
$$

Full details regarding the breakage algorithm for weighted particles including its convergence properties can be found in [25].

\subsection{Particle transport}

At the rate

$$
R_{z, \text { transport }}=\frac{N_{z}(t)}{\tau_{z}}
$$

a particle is uniformly selected in compartment $z$ to undergo the following jump processes which depend on the initial location:

- $z=z_{1}$

$$
\left(z_{1}, x_{i}, w_{i}\right) \rightarrow\left(z_{2}, x_{i}, w_{i}\right)
$$

- $z=z_{3}$

$$
\left(z_{3}, x_{i}, w_{i}\right) \rightarrow\left(z_{2}, x_{i}, w_{i}\right)
$$

- $z=z_{2}$

$$
\begin{aligned}
& \left(z_{2}, x_{i}, w_{i}\right) \rightarrow\left(z_{1}, x_{i}, w_{i}\right), \quad \text { with probability }=0.5 \\
& \left(z_{2}, x_{i}, w_{i}\right) \rightarrow\left(z_{3}, x_{i}, w_{i}\right), \quad \text { with probability }=0.5 .
\end{aligned}
$$

Full details regarding the implementation of particle transport in stochastic particle frameworks can be found in $[52,24,25]$. 


\subsection{Collision}

The outcome of a collision jump process depends on the coalescence efficiency $\widetilde{K}$ :

$$
\begin{aligned}
\left(x_{i}, w_{i}\right),\left(x_{j}, w_{j}\right) \rightarrow\left(T_{\text {comp }}\left(x_{i}+x_{j}\right), \gamma_{\text {coal }}\left(x_{i}, w_{i}, x_{j}, w_{j}\right)\right),\left(x_{j}, w_{j}\right), & \text { if } \widetilde{K}\left(x_{i}, x_{j}\right)=1, \\
\left(x_{i}, w_{i}\right),\left(x_{j}, w_{j}\right) \rightarrow\left(T_{\text {comp }}\left(x_{i}\right), w_{i}\right),\left(x_{j}, w_{j}\right), & \text { if } \widetilde{K}\left(x_{i}, x_{j}\right)=0 .
\end{aligned}
$$

The second particle, $x_{j}$, is left unchanged in order to achieve the correct convergence properties for SWA [25, 27, 26]. The weight transfer function for coalescence $\gamma_{\text {coal }}$ is defined to conserve total mass:

$$
\gamma_{\text {coal }}\left(x_{i}, w_{i}, x_{j}, w_{j}\right)=w_{i} \frac{m\left(x_{i}\right)}{m\left(x_{i}\right)+m\left(x_{j}\right)} .
$$

Other mathematically valid weight transfer functions are investigated in [27].

A key challenge in implementing the collision process is the efficient calculation of the total rate. The collision kernel for the particle pair $\left(x_{i}, w_{i}\right)$ and $\left(x_{j}, w_{j}\right)$ in the context of SWA is [27]:

$$
\begin{aligned}
K_{z, \text { SWA }}\left(x_{i}, w_{i}, x_{j}, w_{j}\right) & =K_{z}\left(x_{i}, x_{j}\right) w_{j} \\
& =\omega_{\text {impeller }} k_{z, \text { col }} C\left(d_{i}, d_{j}\right) w_{j}
\end{aligned}
$$

In order for the rate to be calculated efficiently, it is necessary for the kernel to be in the form:

$$
\begin{gathered}
K_{z, \mathrm{SWA}}\left(x_{i}, w_{i}, x_{j}, w_{j}\right)=h_{1}^{(1)}\left(x_{i}, w_{i}\right) h_{2}^{(1)}\left(x_{j}, w_{j}\right)+h_{1}^{(2)}\left(x_{i}, w_{i}\right) h_{2}^{(2)}\left(x_{j}, w_{j}\right)+ \\
\ldots+h_{1}^{\left(n_{h}\right)}\left(x_{i}, w_{i}\right) h_{2}^{\left(n_{h}\right)}\left(x_{j}, w_{j}\right)
\end{gathered}
$$


so that its sum can be factorised as

$$
\sum_{i \neq j}^{N_{z}(t)} K_{z, \mathrm{SWA}}\left(x_{i}, w_{i}, x_{j}, w_{j}\right)=\lambda_{1}^{(1)} \lambda_{2}^{(1)}-H^{(1)}+\lambda_{1}^{(2)} \lambda_{2}^{(2)}-H^{(2)}+\ldots+\lambda_{1}^{\left(n_{h}\right)} \lambda_{2}^{\left(n_{h}\right)}-H^{\left(n_{h}\right)}
$$

where $\lambda$ and $H$ are defined as

$$
\begin{aligned}
\lambda_{l}^{(k)} & =\sum_{i=1}^{N_{z}(t)} h_{l}^{(k)}\left(x_{i}, w_{i}\right), \quad l=1,2, \quad k=1,2, \ldots, n_{h}, \\
H^{(k)} & =\sum_{i=1}^{N_{z}(t)} h_{1}^{(k)}\left(x_{i}, w_{i}\right) h_{2}^{(k)}\left(x_{i}, w_{i}\right), \quad k=1,2, \ldots, n_{h} .
\end{aligned}
$$

Note that the terms $H^{(k)}$ are subtracted from $\lambda_{1}^{(k)} \lambda_{2}^{(k)}$ to remove self-collisions. In this form, the quantities $h_{l}^{(k)}$ and $h_{1}^{(k)} h_{2}^{(k)}$ can be stored in a binary tree which enable rapid calculation of the sums $\lambda_{l}^{(k)}$ and $H^{(k)}[27,53]$.

The selection of the particle pair $\left(x_{i}, w_{i}\right)$ and $\left(x_{j}, w_{j}\right)$ depends on Equation (50) which can be interpreted as the sum of $n_{h}$ individual rate terms. So, with probability

$$
\frac{\lambda_{1}^{(k)} \lambda_{2}^{(k)}-H^{(k)}}{\sum_{i \neq j}^{N_{z}(t)} K_{z, \text { SWA }}\left(x_{i}, w_{i}, x_{j}, w_{j}\right)}
$$

the index, $i$ and $j$, of the colliding particles are chosen according to the respective probabilities

$$
\frac{h_{1}^{(k)}\left(x_{i}, w_{i}\right)}{\lambda_{1}^{(k)}} \text { and } \frac{h_{2}^{(k)}\left(x_{j}, w_{j}\right)}{\lambda_{2}^{(k)}} \text {, with } i \neq j .
$$

It is determined in Section 3.2 that the EKE collision rate function is the most suitable rate function for the high-shear granulation system. The collision rate function only affects the rate and the collision jump mechanism (46) remains unchanged. 
The collision kernel using the EKE rate function is

$$
K_{z, \mathrm{SWA}}^{\mathrm{EKE}}\left(x_{i}, w_{i}, x_{j}, w_{j}\right)=\omega_{\text {impeller }} k_{z, \text { col }}\left(d_{i}+d_{j}\right)^{2} \sqrt{\frac{1}{d_{i}{ }^{3}}+\frac{1}{d_{j}{ }^{3}}} w_{j} .
$$

The calculation of the rate becomes complicated for this kernel due to its complex form. In order to calculate the exact rate, it is necessary to loop through each pair of particle and this is computationally expensive. Therefore, the majorant technique which provides computationally efficient implementations of the kernel $K$ is applied [26, 27, 28, 29].

To arrive in the form shown by Equation (50), the kernel (55) is estimated by the majorant kernel:

$$
\hat{K}_{z, \mathrm{SWA}}^{\mathrm{EKE}}\left(x_{i}, w_{i}, x_{j}, w_{j}\right)=k_{\text {maj }} \omega_{\text {impeller }} k_{z, \text { col }}\left(d_{i}{ }^{2}+d_{j}{ }^{2}\right)\left(\frac{1}{d_{i}{ }^{1.5}}+\frac{1}{d_{j}{ }^{1.5}}\right) w_{j}
$$

such that $K \leq \hat{K}$ and $k_{\text {maj }}=1.42$ is the majorant scaling factor required to maintain the inequality. The value of $k_{\text {maj }}$ is calculated by determining the maximum point of the ratio $K / \hat{K}$ and solving it such that the ratio equals to one. This ensures that the majorant kernel is as efficient as it could be.

The total majorant collision jump rate in compartment $z$ can then be 
expressed as:

$$
\begin{aligned}
\hat{R}_{z, \text { col }} & =\frac{1}{n_{z}} \sum_{i \neq j}^{N_{z}(t)} \hat{K}_{z, \mathrm{SWA}}^{\mathrm{EKE}}\left(x_{i}, w_{i}, x_{j}, w_{j}\right) \\
& =\frac{k_{\text {maj }} \omega_{\text {impeller }} k_{z, \text { col }}}{n_{z}}\left\{\left[\sum_{i=1}^{N(t)} d_{i}^{0.5} \sum_{j=1}^{N(t)} w_{j}-\sum_{i=1}^{N(t)} d_{i}{ }^{0.5} w_{i}\right]\right. \\
& +\left[\sum_{i=1}^{N(t)} d_{i}{ }^{2} \sum_{j=1}^{N(t)} d_{j}{ }^{-1.5} w_{j}-\sum_{i=1}^{N(t)} d_{i}{ }^{0.5} w_{i}\right]+\left[\sum_{i=1}^{N(t)} d_{i}{ }^{-1.5} \sum_{j=1}^{N(t)} d_{j}{ }^{2} w_{j}-\sum_{i=1}^{N(t)} d_{i}{ }^{0.5} w_{i}\right] \\
+ & {\left.\left[\sum_{i=1}^{N(t)} 1 \sum_{j=1}^{N(t)} d_{j}{ }^{0.5} w_{j}-\sum_{i=1}^{N(t)} d_{i}{ }^{0.5} w_{i}\right]\right\} }
\end{aligned}
$$

Note that the term $\sum d_{i}{ }^{0.5} w_{i}$ is subtracted from the terms to exclude selfcollisions. The particle selection properties and the partial sums for this majorant kernel are summarised in Table 4.

Table 4: Particle selection properties based on Equation (57).

\begin{tabular}{llllll}
\hline$k$ & Equation & $\lambda_{1}^{(k)}$ & $\lambda_{2}^{(k)}$ & $h_{1}^{(k)}\left(x_{i}, w_{i}\right)$ & $h_{2}^{(k)}\left(x_{j}, w_{j}\right)$ \\
\hline 1 & {$\left[\sum d_{i}{ }^{0.5} \sum w_{j}-\sum d_{i}{ }^{0.5} w_{i}\right]$} & $\sum d_{i}{ }^{0.5}$ & $\sum w_{j}$ & $d_{i}{ }^{0.5}$ & $w_{j}$ \\
2 & {$\left[\sum d_{i}{ }^{2} \sum d_{j}{ }^{-1.5} w_{j}-\sum d_{i}{ }^{0.5} w_{i}\right]$} & $\sum d_{i}{ }^{2}$ & $\sum d_{j}{ }^{-1.5} w_{j}$ & $d_{i}{ }^{2}$ & $d_{j}{ }^{-1.5} w_{j}$ \\
3 & {$\left[\sum d_{i}{ }^{-1.5} \sum d_{j}{ }^{2} w_{j}-\sum d_{i}{ }^{0.5} w_{i}\right]$} & $\sum d_{i}{ }^{-1.5}$ & $\sum d_{j}{ }^{2} w_{j}$ & $d_{i}{ }^{-1.5}$ & $d_{j}{ }^{2} w_{j}$ \\
4 & {$\left[\sum 1 \sum d_{j}{ }^{0.5} w_{j}-\sum d_{i}{ }^{0.5} w_{i}\right]$} & $\sum 1$ & $\sum d_{j}{ }^{0.5} w_{j}$ & 1 & $d_{j}{ }^{0.5} w_{j}$ \\
\hline
\end{tabular}

When a majorant kernel is used, it is necessary to introduce fictitious jumps in order to achieve the correct convergence properties [28]. Once the colliding pair $\left(x_{i}, w_{i}\right)$ and $\left(x_{j}, w_{j}\right)$ in a compartment $z$ is chosen according to the selection properties in Table 4, the collision jump process (46) is 
performed with probability

$$
\frac{K_{z, \mathrm{SWA}}^{\mathrm{EKE}}\left(x_{i}, w_{i}, x_{j}, w_{j}\right)}{\hat{K}_{z, \mathrm{SWA}}^{\mathrm{EKE}}\left(x_{i}, w_{i}, x_{j}, w_{j}\right)},
$$

otherwise the jump is fictitious, i.e. nothing happens in this step but time moves forward.

\subsection{Simulation algorithm}

The waiting time $t_{\text {wait }}$ is generated from an exponential distribution [28]

$$
\operatorname{Prob}\left\{t_{\text {wait }} \geq s\right\}=\exp \left(-R_{\text {total }} s\right), \quad s \geq 0,
$$

with the parameter

$$
R_{\text {total }}=\sum_{z=\mathbb{Z}}\left[R_{z, \text { droplet }}+R_{z, \text { break }}+R_{z, \text { transport }}+\hat{R}_{z, \text { col }}\right]
$$

A particle process (inception, breakage, transport, or collision) in a compartment $z$ is chosen according to the probabilities

$$
\frac{R_{z, \text { droplet }}}{R_{\text {total }}}, \quad \frac{R_{z, \text { break }}}{R_{\text {total }}}, \quad \frac{R_{z, \text { transport }}}{R_{\text {total }}}, \frac{\hat{R}_{z, \text { col }}}{R_{\text {total }}} .
$$

$R_{z \text {, droplet }}, R_{z \text {, break }}, R_{z \text {, transport }}$, and $\hat{R}_{z \text {, col }}$ are given by Equations (36), (37), (42), and (57). Since there are three compartments, there are 12 jump processes considered in total. Once a particle process in a specific compartment is selected, the respective jump mechanisms described in Sections 4.1 - 4.4 are performed.

In between jump processes, the components of the particles change according to the following system of ordinary differential equations using the 
Linear Process Deferment Algorithm [54]:

$$
\begin{aligned}
& \frac{\mathrm{d} s_{\mathrm{o}}}{\mathrm{d} t}=0, \quad \frac{\mathrm{d} s_{\mathrm{r}}}{\mathrm{d} t}=r_{z, \text { reac, } \mathrm{e}}(x)+r_{z, \text { reac }, \mathrm{i}}, \\
& \frac{\mathrm{d} l_{\mathrm{e}}}{\mathrm{d} t}=-r_{z, \text { reac, } \mathrm{e}}(x)-r_{z, \text { pen }}(x), \quad \frac{\mathrm{d} l_{\mathrm{i}}}{\mathrm{d} t}=-r_{z, \text { reac }, \mathrm{i}}(x)+r_{z, \text { pen }}(x), \\
& \frac{\mathrm{d} p}{\mathrm{~d} t}=-r_{z, \text { reac }, \mathrm{i}}(x) .
\end{aligned}
$$

In Section 2.1, it is stated that the densities of reacted solid and liquid are assumed to be the same and the main justification of this assumption is that it simplifies the system of ODEs shown because the only contribution to $s_{\mathrm{r}}$ is from $l_{\mathrm{e}}$ and $l_{\mathrm{i}}$. The reaction submodel can be further improved by considering the solubility of lactose in water and the contribution of $s_{\mathrm{o}}$. However this is not the focus of this work and it is decided to keep the model simple by assuming that the densities of reacted solid and liquid are equal.

\section{Application}

The population balance model is simulated as closely as possible to a set of experimental conditions chosen from [30, Experiment B1]. The experimental conditions and several key model parameters are listed in Table 5. For the purpose of validating the population balance model, measurements of particle size distribution [30] and porosities [45] are available from the same set of experimental conditions.

The ability of the population balance model to predict the experimental measurements is investigated in this section, in particular the difference observed when the new compartmental model is used as opposed to using a 
single compartment. Here, the rate parameters described in Section 2.2 are optimised to fit the simulated responses to the experimental measurements using the software MoDS (Model Development Suite) [55]. The model contains five rate parameters $\left(k_{z \text {, break }}, k_{z \text {, reac }}, k_{z \text {, pen }}, k_{z \text {, col }}\right.$, and $\left.k_{z \text {, comp }}\right)$ which are allowed to vary between different compartments. By assuming the rates of the particle processes are the same in $z_{1}$ and $z_{3}$, there are $2 \times 5=10$ parameters to consider for the three-compartment model. This assumption also conforms to the symmetrical geometry of the mixer (Figure 1). For the single-compartment model, there are only 5 parameters to consider.

For each model evaluation, the compartments are initialised with a set of non-porous particles of the form:

$$
x=\left(s_{\mathrm{o}}, 0,0,0,0\right)
$$

following the measured size distribution of the initial lactose powder $\left(\mu_{\mathrm{psd}}=\right.$ $\left.38.93 \mu \mathrm{m}, \sigma_{\mathrm{psd}}=1.6 \mu \mathrm{m}\right)$. Once the solid particles are initialised, liquid droplets of the form

$$
x=\left(0,0, V_{\text {droplet }}=1.8 \times 10^{-13} \mathrm{~m}^{3}, 0,0\right),
$$

are added at the rate defined by Equation (36) and stopped after 120 seconds. The volume of the droplets follows the specification of the nozzles used in the experiments. The simulation is then allowed to continue for 300 seconds after the liquid addition phase and this refers to the wet massing time in Table 5. Each model evaluation consists of a number of independent stochastic runs and the results are averaged over the runs.

The quality of the stochastic simulations depends on the number of computational particles and the number of stochastic runs at the expense of CPU 
time. In a preliminary convergence test, it is determined that $N_{\max }=2048$ particles per compartment and 8 stochastic runs are sufficient to achieve convergence for this system. Using these numbers, a model evaluation takes about a few minutes for the single-compartment model and about an hour for the three-compartment model.

To quantify the agreement between the model and experiment, the sum of squares objective function is used:

$$
O F=\sum_{i=1}^{Y}\left(y_{i}^{\text {sim }}-y_{i}^{\text {exp }}\right)^{2},
$$

where $y^{\text {sim }}$ denotes the model response, $y^{\exp }$ denotes the experimental response and $Y$ is the total number of responses.

Optimisation of the objective function is carried out in two stages. The first stage involves a quasi-random global search using a Sobol low-discrepancy sequence [56]. This is followed by a local optimisation starting from the best point using the Hooke and Jeeves' algorithm [57].

The objective function includes a particle size distribution and also particle porosities. The particle size distribution is represented by mass fractions, $f_{\mathrm{m}}$, of 19 size classes. On the other hand, only porosities for 15 particle size classes are included in the objective function as opposed to 19 due to limitations of the experimental measurements. Considering both sets of data, there are 34 observation points in total.

In order to highlight the importance of considering the heterogeneity of our granulation process, two versions of the population balance model which differ in the number of compartments (one and three) are investigated. The optimisation of the objective function is carried out for both models and the 
Table 5: Model physical parameters.

\begin{tabular}{|c|c|c|}
\hline Description & Symbol & Value \\
\hline \multicolumn{3}{|l|}{ Known process settings } \\
\hline Material density & $\rho_{s_{\mathrm{o}}}$ & $1545.0 \mathrm{~kg} / \mathrm{m}^{3}$ \\
\hline Binder density & $\rho_{l_{\mathrm{e}}}$ & $998.0 \mathrm{~kg} / \mathrm{m}^{3}$ \\
\hline Binder viscosity & $\eta$ & $1.0 \times 10^{-3} \mathrm{~Pa} \mathrm{~s}$ \\
\hline Reactor volume & $V_{\text {reactor }}$ & $3.0 \times 10^{-3} \mathrm{~m}^{3}$ \\
\hline Impeller speed & $\omega_{\text {impeller }}$ & $2 \mathrm{rev} / \mathrm{s}$ \\
\hline Binder flow rate & $\dot{V}_{\mathrm{l}}$ & $1.25 \times 10^{-6} \mathrm{~m}^{3} / \mathrm{s}$ \\
\hline Wet massing time & - & $300 \mathrm{~s}$ \\
\hline Binder to powder ratio & - & $150 \mathrm{ml}: 1000 \mathrm{~g}$ \\
\hline \multicolumn{3}{|l|}{ Model parameters - approximated } \\
\hline Powder size distribution; location & $\mu_{\mathrm{psd}}$ & $38.93 \mu \mathrm{m}$ \\
\hline Powder size distribution; shape & $\sigma_{\mathrm{psd}}$ & $1.6 \mu \mathrm{m}$ \\
\hline Droplet volume & $V_{\text {droplet }}$ & $1.80 \times 10^{-13} \mathrm{~m}^{3}$ \\
\hline Particle-particle collision velocity & $U_{\text {col }}$ & $9.11 \times 10^{-2} \mathrm{~m} / \mathrm{s}$ \\
\hline Minimum particle porosity after compaction & $\epsilon_{\min }$ & 0.25 \\
\hline Breakage; minimum fragment volume & $v_{\text {frag,min }}$ & $5.236 \times 10^{-13} \mathrm{~m}^{3}$ \\
\hline Breakage; particle-impeller impact velocity & $U_{\text {imp }}$ & $0.82 \mathrm{~m} / \mathrm{s}$ \\
\hline
\end{tabular}

optimised rate parameters are shown in Tables 6 and 7. For both models, a total of 10,000 Sobol evaluations and a few hundred additional evaluations for the local optimisation are performed to arrive at the optimised rate parameters. 
Figures 10 and 11 show the best fit size distribution and porosities respectively from the population balance model. Looking closely at Figure 10, it can be observed that the multi-compartment model is able to describe the process more accurately as the generated size distribution is much closer to the experimental data. By using three compartments instead of one, the model is able to generate a wider range of particle sizes compared to the size independent kernel.

As for the porosities (Figure 11), the value 0 is given when there are no particles of that size. Similar to the size distribution, the values generated with the multi-compartment model are also much closer to the experimental data partly because the model is not able to generate any particles above $850 \mu \mathrm{m}$ with a single compartment. It may be observed that the confidence interval for the porosity of particles with the size $850 \mu \mathrm{m}$ is very large but this is should not be a concern because there are very few particles in this size class as shown in the corresponding size distribution.

Table 6: Optimised rate parameters and objective function when the single-compartment model is used.

\begin{tabular}{ccc}
\hline Rate parameter & Units & Value \\
\hline$k_{z, \text { break }}$ & $\mathrm{s} \mathrm{m}^{-5}$ & $2.07 \times 10^{5}$ \\
$k_{z, \text { reac }}$ & $\mathrm{m} \mathrm{s}^{-1}$ & $3.17 \times 10^{-11}$ \\
$k_{z, \text { pen }}$ & $\mathrm{kg}^{0.5} \mathrm{~s}^{-1.5} \mathrm{~m}^{-3.5}$ & $3.77 \times 10^{3}$ \\
$k_{z, \text { col }}$ & $\mathrm{m}^{3}$ & $5.37 \times 10^{-13}$ \\
$k_{z, \text { comp }}$ & - & $5.24 \times 10^{-2}$ \\
\hline
\end{tabular}

Objective function

1900 
Table 7: Optimised rate parameters and objective function when the multi-compartment model is used.

\begin{tabular}{cccc}
\hline Rate parameter & Units & $z_{1} / z_{3}$ & $z_{2}$ \\
\hline$k_{z, \text { break }}$ & $\mathrm{s} \mathrm{m}^{-5}$ & $5.18 \times 10^{10}$ & $3.56 \times 10^{3}$ \\
$k_{z, \text { reac }}$ & $\mathrm{m} \mathrm{s}^{-1}$ & $1.21 \times 10^{-16}$ & $2.10 \times 10^{-14}$ \\
$k_{z, \text { pen }}$ & $\mathrm{kg}^{0.5} \mathrm{~s}^{-1.5} \mathrm{~m}^{-3.5}$ & $8.85 \times 10^{3}$ & $1.22 \times 10^{1}$ \\
$k_{z, \text { col }}$ & $\mathrm{m}^{3}$ & $7.60 \times 10^{-15}$ & $2.77 \times 10^{-9}$ \\
$k_{z, \text { comp }}$ & - & $1.00 \times 10^{0}$ & $3.61 \times 10^{-1}$ \\
\hline
\end{tabular}

Objective function

326

\section{Conclusions}

This paper presents how DEM simulations can be coupled to a population balance model to model a batch granulation process. As both approaches have different capabilities, they are used to complement each other in this work.

DEM is used to analyse flow patterns in the mixer and the simulations reveal two crucial findings. First, it is found that segregation of particles is inherent especially in the axial direction and this justifies the usage of multiple compartments in the population balance model. Second, the results suggest that the collision frequencies between particles are size dependent and this should be reflected in the population balance model. Even though DEM is able to provide detailed information regarding the movement of particles, it is computationally expensive and unsuitable for long time scale studies. 
On the other hand, population balance models are computationally cheap. However, they often require some prior knowledge of the system. For example, the residence times of the multi-compartment model and the rate of collision between particles are unknown. Hence, methodologies are developed to determine the compartmental residence times and also a suitable collision rate function for the population balance model from DEM.

Based on the particle-particle collision frequencies extracted from DEM, it is determined that the EKE kernel is the most appropriate kernel to use in the population balance model. In order to use it in a stochastic particle

framework, the majorant technique is adapted to this kernel to enable rapid calculation of the collision rate.

Then, the performance of the multi-compartment model developed in this paper is assessed by comparing the generated particle size distributions and porosities with a set of experimental data. Our results suggest that it is important to consider the heterogeneous behaviour of powder mixing processes in order to achieve good agreement with our experimental data.

Despite the improvement observed, there are noticeable weaknesses in the model especially in predicting the properties of the larger particles. Hence, an immediate future work will involve a detailed sensitivity analysis to decompose the effects of the model parameters on the model responses. This should reveal further insights on the behaviour of the model.

\section{Acknowledgements}

This project is partly funded by the National Research Foundation (NRF), Prime Minister's Office, Singapore under its Campus for Research Excellence 
and Technological Enterprise (CREATE) Programme.

\section{Appendix A. Derivation of three compartment mixing model}

A model for each particle volume fraction evolution in each compartment is derived by considering an idealised reactor system as shown in Figure A.12. Here, a tertiary particle colour system is initially loaded as [red, green, blue] and each compartment is perfectly mixed. Such a construction assumes that the separation between compartment boundaries is sufficiently large and the time step between analysis frames is sufficiently small such that particles cannot 'skip' over compartments (e.g. from $C_{1}$ directly to $C_{3}$ ). The volume flow rate from compartment $k$ to $p$ can then be written as the sum of the time-dependent component volume flow-rates $Q_{k \rightarrow p}^{[j]}(t)$ as:

$$
Q_{k \rightarrow p}=Q_{k \rightarrow p}^{[\text {red] }}(t)+Q_{k \rightarrow p}^{[\text {green] }}(t)+Q_{k \rightarrow p}^{[\text {blue }]}(t) .
$$

Since the mixer is being operated at a constant speed we expect the volume transfer rate (driven by the energy supplied by the motor) to be constant with respect to time (neglecting the time taken to establish any potential particle flow channels). Assuming constant volume hold-up within each compartment (no net movement between compartments was observed during the experiment) it follows that, for compartments connected to a single other compartment (compartment 1 and 3 in Figure A.12),

$$
Q_{k \rightarrow p}=Q_{p \rightarrow k} .
$$

Furthermore, mixer symmetry dictates that:

$$
Q_{1 \rightarrow 2}=Q_{3 \rightarrow 2},
$$


and thus:

$$
Q_{1 \rightarrow 2}=Q_{2 \rightarrow 1}=Q_{2 \rightarrow 3}=Q_{3 \rightarrow 2} .
$$

If particles have a uniform density across the system then the corresponding mass flow rates $\dot{M}$ may be written as:

$$
\dot{M}_{1 \rightarrow 2}=\dot{M}_{2 \rightarrow 1}=\dot{M}_{2 \rightarrow 3}=\dot{M}_{3 \rightarrow 2} .
$$

By definition the average residence time $\tau$ of a compartment is given by:

$$
\tau_{i}=\frac{V_{i, T}}{Q_{i, \text { out }}},
$$

where $V_{i, T}$ and $Q_{i, \text { out }}$ are the total particle volume and volumetric flow-rate out of compartment $i$, respectively. The compartments are defined such that $V_{1, T}=V_{2, T}=V_{3, T}$ and $Q_{1, \text { out }}=Q_{2, \text { out }} / 2=Q_{3, \text { out }}$, and so:

$$
\tau_{1}=2 \tau_{2}=\tau_{3}
$$

Again, assuming constant particle density, a dynamic component balance over the red particles in $C_{1}$ may be written as:

$$
\frac{\mathrm{d} V_{1, \text { red }}(t)}{\mathrm{d} t}=Q_{2 \rightarrow 1}^{[\mathrm{red}]}-Q_{1 \rightarrow 2}^{[\mathrm{red}]}
$$

If half of the red particles in $C_{2}$ are assumed to exit into $C_{1}$ and half through to $C_{3}$ (again this assumes that $C_{2}$ is well mixed) then it follows from (A.8) that:

$$
\begin{aligned}
V_{1, T} \frac{\mathrm{d} \hat{\nu}_{1, \text { red }}(t)}{\mathrm{d} t} & =\frac{V_{2, \text { red }}}{2 \tau_{2}}-\frac{V_{1, \text { red }}}{\tau_{1}} \\
\rightarrow \frac{\mathrm{d} \hat{\nu}_{1, \text { red }}(t)}{\mathrm{d} t} & =\frac{1}{\tau_{1}}\left(\hat{\nu}_{2, \text { red }}(t)-\hat{\nu}_{1, \text { red }}(t)\right) .
\end{aligned}
$$


A similar argument applied to $C_{2}$ and $C_{3}$ yields:

$$
\begin{aligned}
& \frac{\mathrm{d} \hat{\nu}_{2, \text { red }}(t)}{\mathrm{d} t}=\frac{1}{\tau_{1}}\left(\hat{\nu}_{1, \text { red }}(t)+\hat{\nu}_{3, \text { red }}(t)-2 \hat{\nu}_{2, \text { red }}(t)\right), \\
& \frac{\mathrm{d} \hat{\nu}_{3, \text { red }}(t)}{\mathrm{d} t}=\frac{1}{\tau_{1}}\left(\hat{\nu}_{2, \text { red }}(t)-\hat{\nu}_{3, \text { red }}(t)\right) .
\end{aligned}
$$

Equations (A.9), (A.10) and (A.11) form a system of ordinary differential equations with initial conditions $\hat{\nu}_{1 \text {,red }}(0)=1$ and $\hat{\nu}_{2 \text {,red }}(0)=\hat{\nu}_{3 \text {,red }}(0)=0$. The solutions to the system are then:

$$
\begin{aligned}
& \hat{\nu}_{1, \text { red }}(t)=\frac{1}{2} \exp \left(-\frac{t}{\tau_{1}}\right)+\frac{1}{6} \exp \left(-\frac{3 t}{\tau_{1}}\right)+\frac{1}{3}, \\
& \hat{\nu}_{2, \text { red }}(t)=-\frac{1}{3} \exp \left(-\frac{3 t}{\tau_{1}}\right)+\frac{1}{3}, \\
& \hat{\nu}_{3, \text { red }}(t)=-\frac{1}{2} \exp \left(-\frac{t}{\tau_{1}}\right)+\frac{1}{6} \exp \left(-\frac{3 t}{\tau_{1}}\right)+\frac{1}{3} .
\end{aligned}
$$

Due to the symmetrical nature of both the mixture operation and loading it is known that in an ideal system:

$$
\begin{aligned}
& \hat{\nu}_{1, \text { blue }}(t)=\hat{\nu}_{3, \text { red }}(t), \\
& \hat{\nu}_{2, \text { blue }}(t)=\hat{\nu}_{2 \text {,red }}(t), \\
& \hat{\nu}_{3, \text { blue }}(t)=\hat{\nu}_{1, \text { red }}(t) .
\end{aligned}
$$

Furthermore, from the definition of volume fraction we have:

$$
\hat{\nu}_{i, \text { green }}(t)=1-\hat{\nu}_{i, \text { red }}(t)-\hat{\nu}_{i, \text { blue }}(t)
$$

for each compartment $i$. Again acknowledging the mixer symmetry, it follows that:

$$
\begin{aligned}
& \hat{\nu}_{1, \text { green }}(t)=\hat{\nu}_{3, \text { green }}(t)=\hat{\nu}_{2, \text { red }}(t)=\hat{\nu}_{2, \text { blue }}(t), \\
& \hat{\nu}_{2, \text { green }}(t)=\frac{2}{3} \exp \left(-\frac{3 t}{\tau_{1}}\right)+\frac{1}{3}
\end{aligned}
$$




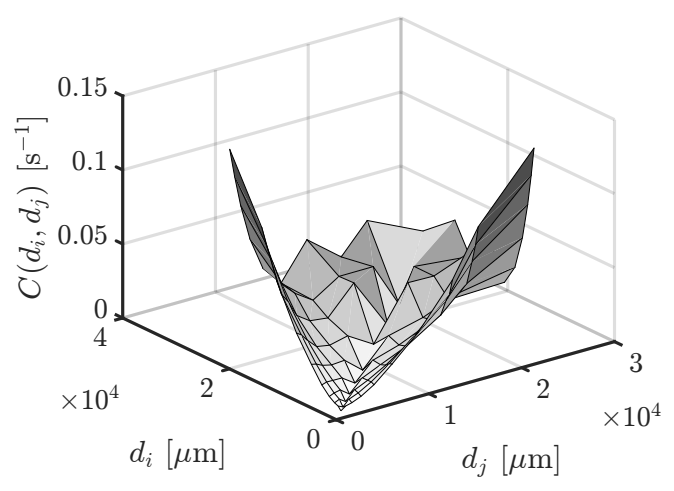

(a) $D E M$

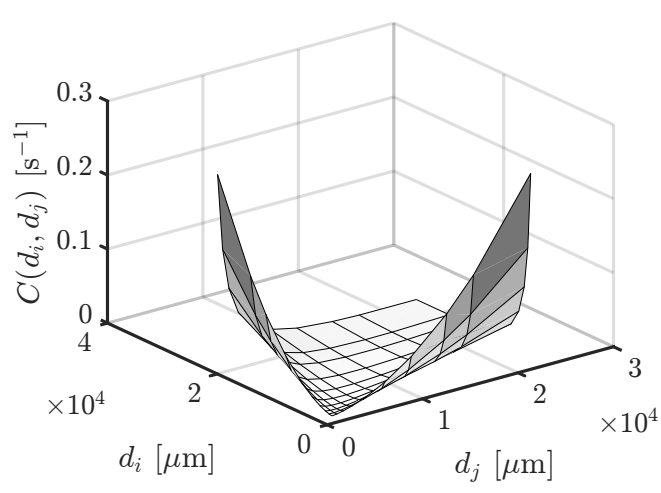

(c) Equipartition of kinetic energy

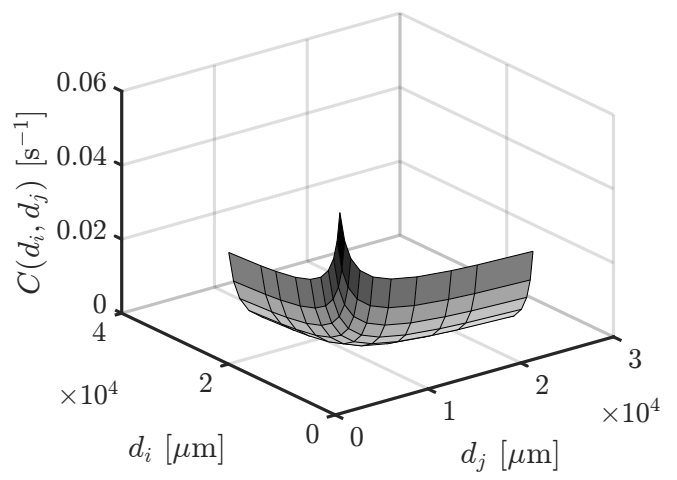

(e) Perikinetic



(b) Size independent

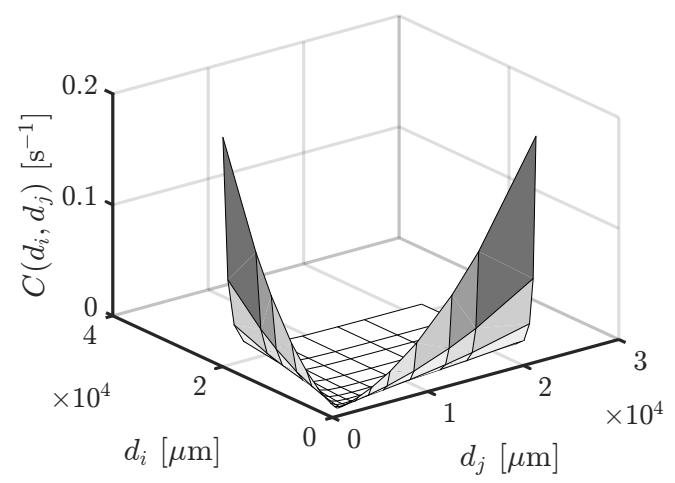

(d) Equipartition of translational momentum



(f) Induced shear

Figure 8: Normalised collision frequencies. 


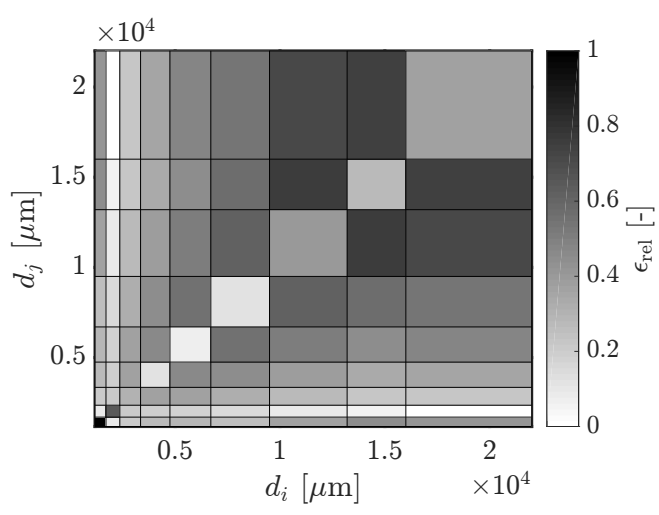

(a) EKE error

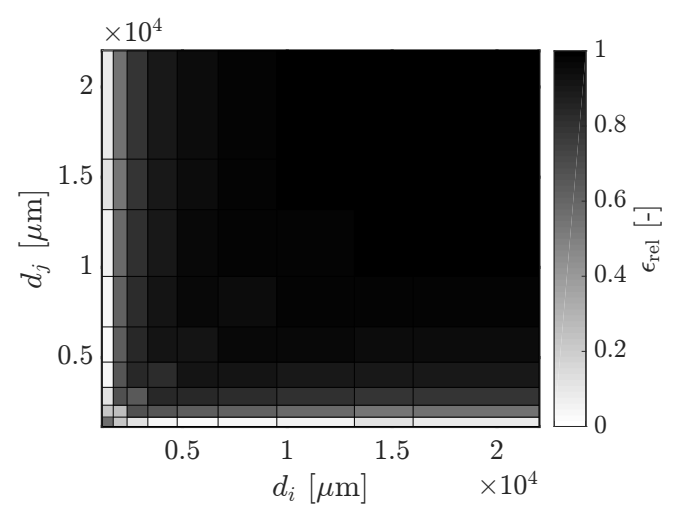

(b) ETM error

Figure 9: Relative error between the DEM extracted frequencies with the two closest collision rate functions - EKE and ETM.

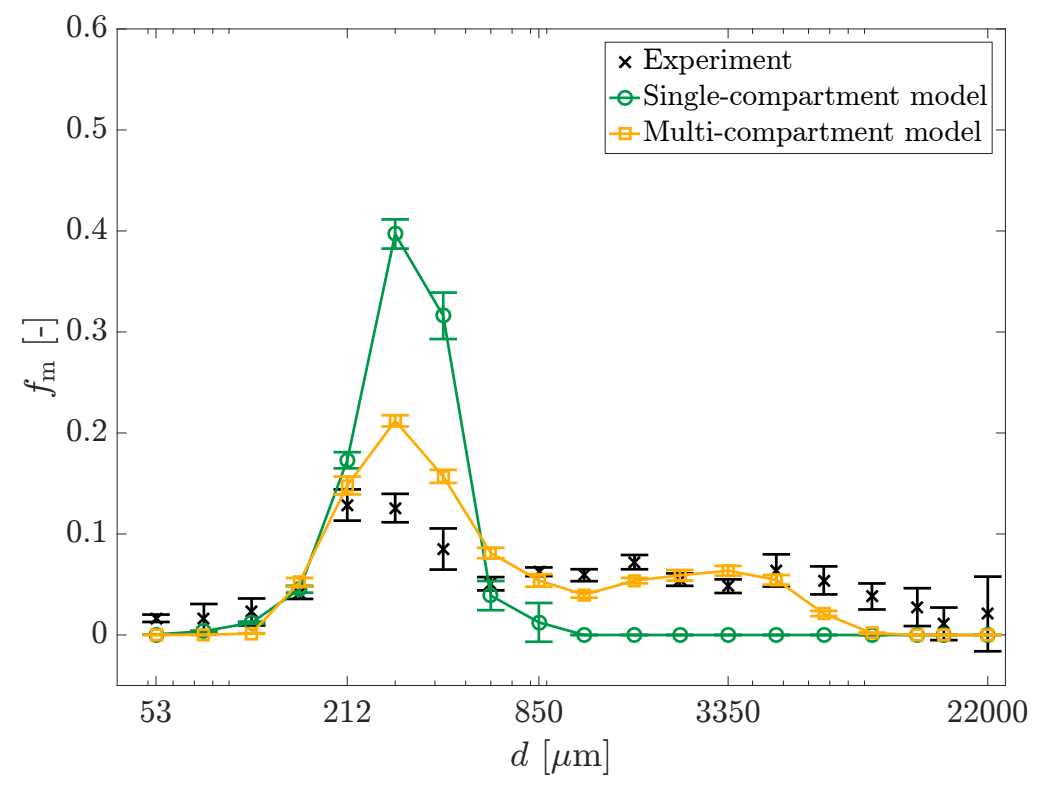

Figure 10: Best fit particle size distributions. 


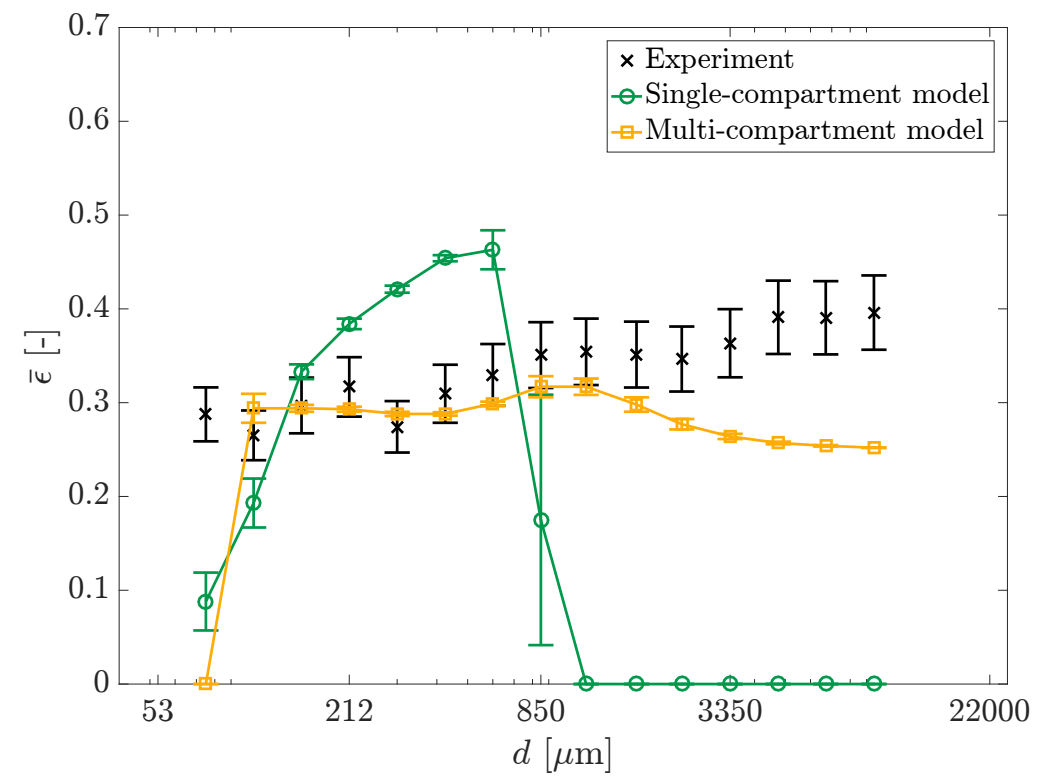

Figure 11: Best fit porosity distributions.

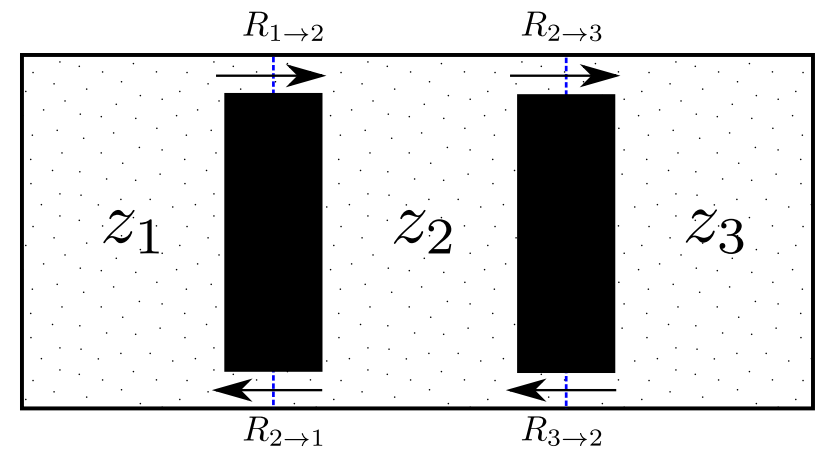

Figure A.12: Idealised compartmental mixer in which particles travel at a constant rate through pre-defined channels. Each compartment acts as a well-mixed CSTR. 
[1] J. N. Michaels, Toward rational design of powder processes, Powder Technology 138 (1) (2003) 1-6, world Congress of Particle Technology. doi:10.1016/j.powtec.2003.08.035.

[2] S. Heinrich, M. Dosta, S. Antonyuk, Chapter two - multiscale analysis of a coating process in a wurster fluidized bed apparatus, in: G. B. Marin, J. Li (Eds.), Mesoscale Modeling in Chemical Engineering Part I, Vol. 46 of Advances in Chemical Engineering, Academic Press, 2015, pp. 83 - 135. doi:10.1016/bs.ache.2015.10.012.

[3] S. M. Iveson, J. D. Litster, K. Hapgood, B. J. Ennis, Nucleation, growth and breakage phenomena in agitated wet granulation processes: a review, Powder Technology 117 (1-2) (2001) 3-39, granulation and Coating of Fine Powders. doi:10.1016/S0032-5910(01)00313-8.

[4] A. Braumann, M. J. Goodson, M. Kraft, P. R. Mort, Modelling and validation of granulation with heterogeneous binder dispersion and chemical reaction, Chemical Engineering Science 62 (17) (2007) 4717-4728. doi:10.1016/j.ces.2007.05.028.

[5] S. M. Iveson, Limitations of one-dimensional population balance models of wet granulation processes, Powder Technology 124 (3) (2002) 219-229, control of Particulate Processess IV. doi:10.1016/S0032-5910(02)000268.

[6] M. Oullion, G. Reynolds, M. Hounslow, Simulating the early stage of high-shear granulation using a two-dimensional montecarlo approach, Chemical Engineering Science 64 (4) (2009) $673-$ 
685, 3rd International Conference on Population Balance Modelling. doi:10.1016/j.ces.2008.08.014.

[7] C. L. Marshall Jr., P. Rajniak, T. Matsoukas, Multi-component population balance modeling of granulation with continuous addition of binder, Powder Technology 236 (2013) 211-220, special Issue: Pharmaceutical Powders. doi:10.1016/j.powtec.2012.01.027.

[8] D. Barrasso, R. Ramachandran, Multi-scale modeling of granulation processes: Bi-directional coupling of PBM with DEM via collision frequencies, Chemical Engineering Research and Designdoi:10.1016/j.cherd.2014.04.016.

[9] A. Chaudhury, H. Wu, M. Khan, R. Ramachandran, A mechanistic population balance model for granulation processes: Effect of process and formulation parameters, Chemical Engineering Science 107 (2014) 76-92. doi:10.1016/j.ces.2013.11.031.

[10] A. Darelius, H. Brage, A. Rasmuson, I. N. Björn, S. Folestad, A volumebased multi-dimensional population balance approach for modelling high shear granulation, Chemical Engineering Science 61 (8) (2006) 24822493. doi:10.1016/j.ces.2005.11.016.

[11] J. M.-H. Poon, C. D. Immanuel, F. J. Doyle, III, J. D. Litster, A threedimensional population balance model of granulation with a mechanistic representation of the nucleation and aggregation phenomena, Chemical Engineering Science 63 (5) (2008) 1315 - 1329, control of Particulate Processes. doi:10.1016/j.ces.2007.07.048. 
[12] L. X. Liu, J. D. Litster, Population balance modelling of granulation with a physically based coalescence kernel, Chemical Engineering Science 57 (12) (2002) 2183-2191, population balance modelling of particulate systems. doi:10.1016/S0009-2509(02)00110-0.

[13] R. Ramachandran, C. D. Immanuel, F. Stepanek, J. D. Litster, F. J. D. III, A mechanistic model for breakage in population balances of granulation: Theoretical kernel development and experimental validation, Chemical Engineering Research and Design 87 (4) (2009) 598 - 614, 13th European Conference on Mixing: New developments towards more efficient and sustainable operations. doi:10.1016/j.cherd.2008.11.007.

[14] I. Cameron, F. Wang, C. Immanuel, F. Stepanek, Process systems modelling and applications in granulation: A review, Chemical Engineering Science 60 (14) (2005) 3723-3750, granulation across the length scales - 2nd International Workshop on Granulation. doi:10.1016/j.ces.2005.02.004.

[15] B. Freireich, J. Li, J. Litster, C. Wassgren, Incorporating particle flow information from discrete element simulations in population balance models of mixer-coaters, Chemical Engineering Science 66 (16) (2011) 3592-3604. doi:10.1016/j.ces.2011.04.015.

[16] J. Bouffard, F. Bertrand, J. Chaouki, A multiscale model for the simulation of granulation in rotor-based equipment, Chemical Engineering Science 81 (2012) 106-117. doi:10.1016/j.ces.2012.06.025.

[17] J. Li, B. Freireich, C. Wassgren, J. D. Litster, A general compartment- 
based population balance model for particle coating and layered granulation, AIChE Journal 58 (5) (2012) 1397-1408. doi:10.1002/aic.12678.

[18] M. Sen, R. Ramachandran, A multi-dimensional population balance model approach to continuous powder mixing processes, Advanced Powder Technology 24 (1) (2013) 51 - 59. doi:10.1016/j.apt.2012.02.001.

[19] A. Chaudhury, M. E. Armenante, R. Ramachandran, Compartment based population balance modeling of a high shear wet granulation process using data analytics, Chemical Engineering Research and Design 95 (0) (2015) 211-228. doi:10.1016/j.cherd.2014.10.024.

[20] J. A. Gantt, I. T. Cameron, J. D. Litster, E. P. Gatzke, Determination of coalescence kernels for high-shear granulation using DEM simulations, Powder Technology 170 (2) (2006) 53-63. doi:10.1016/j.powtec.2006.08.002.

[21] H. Tan, M. Goldschmidt, R. Boerefijn, M. Hounslow, A. Salman, J. Kuipers, Building population balance model for fluidized bed melt granulation: lessons from kinetic theory of granular flow, Powder Technology 142 (2-3) (2004) 103 - 109. doi:10.1016/j.powtec.2004.04.030.

[22] D. Barrasso, A. Tamrakar, R. Ramachandran, A reduced order PBMANN model of a multi-scale PBM-DEM description of a wet granulation process, Chemical Engineering Science 119 (0) (2014) 319-329. doi:10.1016/j.ces.2014.08.005.

[23] D. Barrasso, T. Eppinger, F. E. Pereira, R. Aglave, K. Debus, S. K. Bermingham, R. Ramachandran, A multi-scale, mechanistic model of 
a wet granulation process using a novel bi-directional PBM-DEM coupling algorithm, Chemical Engineering Science 123 (0) (2015) 500-513. doi:10.1016/j.ces.2014.11.011.

[24] K. F. Lee, S. Mosbach, M. Kraft, W. Wagner, A multicompartment population balance model for high shear granulation, Computers \& Chemical Engineering 75 (2015) 1-13. doi:10.1016/j.compchemeng.2015.01.009.

[25] K. F. Lee, R. I. Patterson, W. Wagner, M. Kraft, Stochastic weighted particle methods for population balance equations with coagulation, fragmentation and spatial inhomogeneity, Journal of Computational Physics 303 (2015) 1-18. doi:10.1016/j.jcp.2015.09.031.

[26] W. J. Menz, R. I. Patterson, W. Wagner, M. Kraft, Application of stochastic weighted algorithms to a multidimensional silica particle model, Journal of Computational Physics 248 (0) (2013) 221-234. doi:10.1016/j.jcp.2013.04.010.

[27] R. I. Patterson, W. Wagner, M. Kraft, Stochastic weighted particle methods for population balance equations, Journal of Computational Physics 230 (19) (2011) 7456 - 7472. doi:10.1016/j.jcp.2011.06.011.

[28] M. Goodson, M. Kraft, An efficient stochastic algorithm for simulating nano-particle dynamics, Journal of Computational Physics 183 (1) (2002) 210-232. doi:10.1006/jcph.2002.7192.

[29] A. Eibeck, W. Wagner, Approximative solution of the coagulation-fragmentation equation by stochastic particle sys- 
tems, Stochastic analysis and applications 18 (6) (2000) 921-948. doi:10.1080/07362990008809704.

[30] C. A. Kastner, G. P. Brownbridge, S. Mosbach, M. Kraft, Impact of powder characteristics on a particle granulation model, Chemical Engineering Science 97 (0) (2013) 282-295. doi:10.1016/j.ces.2013.04.032.

[31] C. Broadbent, J. Bridgwater, D. Parker, The effect of fill level on powder mixer performance using a positron camera, The Chemical Engineering Journal and the Biochemical Engineering Journal 56 (3) (1995) 119-125. doi:10.1016/0923-0467(94)02906-7.

[32] J. Jones, J. Bridgwater, A case study of particle mixing in a ploughshare mixer using positron emission particle tracking, International Journal of Mineral Processing 53 (1) (1998) 29-38. doi:10.1016/S03017516(97)00054-9.

[33] J. Jones, D. Parker, J. Bridgwater, Axial mixing in a ploughshare mixer, Powder Technology 178 (2) (2007) 73-86. doi:10.1016/j.powtec.2007.04.006.

[34] A. Faure, P. York, R. Rowe, Process control and scale-up of pharmaceutical wet granulation processes: a review, European Journal of Pharmaceutics and Biopharmaceutics 52 (3) (2001) 269-277. doi:10.1016/S09396411(01)00184-9.

[35] G. Reynolds, C. Biggs, A. Salman, M. Hounslow, Non-uniformity of binder distribution in high-shear granulation, Powder Technology 140 (3) (2004) 203 - 208, 1st International Workshop on Granulation 
(Granulation across the length scales: linking microscopic experiments and models to real process operation). doi:10.1016/j.powtec.2004.01.017.

[36] A. Braumann, M. Kraft, W. Wagner, Numerical study of a stochastic particle algorithm solving a multidimensional population balance model for high shear granulation, Journal of Computational Physics 229 (20) (2010) 7672-7691. doi:10.1016/j.jcp.2010.06.021.

[37] A. Braumann, M. Kraft, P. R. Mort, Parameter estimation in a multidimensional granulation model, Powder Technology 197 (3) (2010) 196210. doi:10.1016/j.powtec.2009.09.014.

[38] A. Braumann, P. L. W. Man, M. Kraft, Statistical approximation of the inverse problem in multivariate population balance modeling, Industrial \& Engineering Chemistry Research 49 (1) (2010) 428-438. doi:10.1021/ie901230u.

[39] A. Braumann, M. Kraft, Incorporating experimental uncertainties into multivariate granulation modelling, Chemical Engineering Science 65 (3) (2010) 1088-1100. doi:10.1016/j.ces.2009.09.063.

[40] A. Braumann, P. L. W. Man, M. Kraft, The inverse problem in granulation modeling-two different statistical approaches, AIChE Journal 57 (11) (2011) 3105-3121. doi:10.1002/aic.12526.

[41] J. G. Zadow, Lactose: Properties and uses, Journal of Dairy Science 67 (11) (1984) 2654-2679. doi:10.3168/jds.S0022-0302(84)81625-2.

[42] B. J. Ennis, G. Tardos, R. Pfeffer, A microlevel-based characterization of granulation phenomena, Powder Technology 65 (1-3) (1991) 257-272, 
a Special Volume Devoted to the Second Symposium on Advances in Particulate Technology. doi:10.1016/0032-5910(91)80189-P.

[43] M. Dosta, S. Antonyuk, S. Heinrich, Multiscale simulation of the fluidized bed granulation process, Chemical Engineering \& Technology 35 (8) (2012) 1373-1380. doi:10.1002/ceat.201200075.

[44] B. Crüger, V. Salikov, S. Heinrich, S. Antonyuk, V. Sutkar, N. Deen, J. Kuipers, Coefficient of restitution for particles impacting on wet surfaces: An improved experimental approach, Particuology 25 (2016) 1-9. doi:10.1016/j.partic.2015.04.002.

[45] J.-G. Rosenboom, S. Antonyuk, S. Heinrich, M. Kraft, Characterisation of lactose powder and granules for multivariate wet granulation modelling, Chemical Engineering Science 123 (2015) 395 - 405. doi:10.1016/j.ces.2014.11.013.

[46] R. Boerefijn, Z. Ning, M. Ghadiri, Disintegration of weak lactose agglomerates for inhalation applications, International Journal of Pharmaceutics 172 (1-2) (1998) 199-209. doi:10.1016/S0378-5173(98)00207-5.

[47] M. Hounslow, The population balance as a tool for understanding particle rate processes, KONA Powder and Particle Journal 16 (1998) 179193. doi:10.14356/kona.1998021.

[48] S. Rjasanow, W. Wagner, A stochastic weighted particle method for the boltzmann equation, Journal of Computational Physics 124 (2) (1996) 243-253. doi:10.1006/jcph.1996.0057. 
[49] H. Zhao, F. E. Kruis, C. Zheng, A differentially weighted Monte Carlo method for two-component coagulation, Journal of Computational Physics 229 (19) (2010) 6931 - 6945. doi:10.1016/j.jcp.2010.05.031.

[50] R. I. Patterson, W. Wagner, A stochastic weighted particle method for coagulation-advection problems, SIAM Journal on Scientific Computing 34 (3) (2012) B290-B311. doi:10.1137/110843319.

[51] J. R. Norris, Smoluchowski's coagulation equation: uniqueness, nonuniqueness and a hydrodynamic limit for the stochastic coalescent, The Annals of Applied Probability 9 (1) (1999) 78-109. doi:10.1214/aoap/1029962598.

[52] W. J. Menz, J. Akroyd, M. Kraft, Stochastic solution of population balance equations for reactor networks, Journal of Computational Physics 256 (0) (2014) 615-629. doi:10.1016/j.jcp.2013.09.021.

[53] M. Goodson, M. Kraft, Simulation of coalescence and breakage: an assessment of two stochastic methods suitable for simulating liquid-liquid extraction, Chemical Engineering Science 59 (18) (2004) 3865-3881. doi:10.1016/j.ces.2004.05.029.

[54] R. Patterson, J. Singh, M. Balthasar, M. Kraft, J. Norris, The Linear Process Deferment Algorithm: A new technique for solving population balance equations, SIAM Journal on Scientific Computing 28 (1) (2006) 303-320. doi:10.1137/040618953.

[55] CMCL Innovations, MoDS (Model Development Suite), date accessed: 
12.01.2015. (2015).

URL http://www.cmclinnovations.com/mods/

[56] I. M. Sobol, On the systematic search in a hypercube, SIAM Journal on Numerical Analysis 16 (5) (1979) 790-793.

URL http://www.jstor.org/stable/2156633

[57] R. Hooke, T. A. Jeeves, "Direct Search" solution of numerical and statistical problems, Journal of the ACM 8 (2) (1961) 212-229. doi:10.1145/321062.321069. 